\title{
COEXISTENCE FOR A CATALYTIC SURFACE REACTION MODEL
}

\author{
By Maury Bramson ${ }^{1}$ and Claudia NeUhauser ${ }^{2}$ \\ University of Wisconsin and University of Minnesota
}

\begin{abstract}
We consider a two-dimensional catalytic surface reaction between $X$ and $Y_{n}$ with $Y_{n}+n X \rightarrow n X Y$, where $Y_{n}$ is a polymer consisting of $n$. identical atoms, each denoted by $Y$, and $X$ is a monomer. The reactants $X$ and $Y_{n}$ are present above the surface in a gaseous phase, and bond to the surface at certain rates. The resulting atoms $X$ and $Y$ on the surface react if they are sufficiently close to each other; the product $X Y$ then leaves the surface. A classical example is the oxidation of carbon monoxide on a platinum surface. In this case, $n=2, X=\mathrm{CO}$ and $Y_{2}=\mathrm{O}_{2}$. We consider the case in which the polymer consists of $n=N^{2}$ atoms, arranged in a square of length $N$, with $N$ large. We show that when the range of interaction is large compared to the polymer size, $X$ and $Y$ will typically coexist on the catalytic surface for appropriate bonding rates. If, however, the range of interaction is small compared to the polymer size, then, irrespective of the bonding rates, the surface will eventually be fully occupied by the monomer $X$.
\end{abstract}

1. Introduction. An important chemical reaction from automobileemission control involves the oxidation of carbon monoxide on platinum, which serves as a catalytic surface. Carbon monoxide $(\mathrm{CO})$ and oxygen $\left(\mathrm{O}_{2}\right)$ are both observed to adsorb on the surface. Upon adsorption, $\mathrm{O}_{2}$ disassociates into two single oxygen atoms, with each occupying a separate site on the surface; carbon monoxide requires only a single site for adsorption. When $\mathrm{CO}$ and $O$ are sufficiently close to each other on the surface, they react with each other to form carbon dioxide $\left(\mathrm{CO}_{2}\right)$, which then desorbs from the surface, leaving two vacant sites behind. A mathematical model of this reaction was introduced by Ziff, Gulari and Barshad (1986). They replaced the surface of the catalyst by the two-dimensional integer lattice. $\mathrm{CO}$ and $\mathrm{O}_{2}$ molecules were assumed to adsorb immediately after colliding with the vacant sites on the surface. The following important qualitative feature of the model was kept: $\mathrm{O}_{2}$ required an adjacent pair of vacant sites, whereas $\mathrm{CO}$ required a single vacant site for adsorption. Upon landing, $\mathrm{O}_{2}$ was assumed to disassociate into two single oxygen atoms, each occupying a single site. Furthermore, carbon monoxide adjacent to an oxygen atom was assumed to react instantly

\footnotetext{
Received September 1995; revised December 1996.

${ }^{1}$ Research partially supported by NSF Grant DMS-96-26196.

${ }^{2}$ Research partially supported by NSF Grant DMS-94-03644 and Alfred P. Sloan Research Fellowship.

AMS 1991 subject classifications. 60K35, 82C20, 82C22.

Key words and phrases. Interacting particle systems, catalytic surface reaction, phase transition, rescaling.
} 
to form carbon dioxide, which then desorbed from the surface, leaving two vacant adjacent sites behind.

We will refer to this monomer-dimer model as the ZGB model. Using computer simulations, Ziff, Gulari and Barshad investigated this system starting from all sites vacant. The parameters were chosen so that the molecule colliding with the surface would be carbon monoxide (CO) with probability $p$ and oxygen $\left(\mathrm{O}_{2}\right)$ with probability $1-p$ ( $p$ can be thought of as the mole fraction of $\mathrm{CO}$ in the gaseous phase). Their simulations showed that the system undergoes two phase transitions with three different phases:

$$
\begin{aligned}
& p \in\left(0, p_{1}\right] \text { with } p_{1} \approx 0.389 \text { oxygen poisoning, } \\
& p \in\left(p_{1}, p_{2}\right] \text { with } p_{2} \approx 0.525 \text { coexistence, } \\
& p \in\left(p_{2}, 1\right] \text { carbon monoxide poisoning. }
\end{aligned}
$$

(If $p=0$, oxygen continues to bond to the surface until no more vacant pairs of adjacent sites are present.) Here and later on, poisoning will mean that any finite square will eventually be completely occupied by only a single type, in which case the reaction ceases there. Coexistence will mean that for any $\varepsilon>0$ and large enough $L$ (depending on $\varepsilon$ ), any $L \times L$ square will have both types of atoms with probability at least $1-\varepsilon$ at all large enough times (depending on the square). This is equivalent to large squares possessing vacant sites, and hence implies that the reaction will continue indefinitely. A quite interesting aspect of the ZGB model is the following: the simulations strongly indicate that the process exhibits a first-order phase transition at $p_{2}$, with the density of $\mathrm{CO}$ in equilibrium jumping from a little less than 0.25 to 1.0. The transition at $p_{1}$, however, appears to be continuous. (A phase diagram adapted from Ziff, Gulari and Barshad is given in Figure 1.)

The paper by Ziff, Gulari and Barshad has sparked considerable interest in the physics literature. In a series of nonrigorous papers, the behavior of the ZGB model was more closely examined [see, e.g., Dickman (1986), Evans and Miesch (1991) and Jensen, Fogedby and Dickman (1990)]. In the mathematics literature, a few rigorous results for variations of the ZGB model are known. Grannan and Swindle (1990) assumed that oxygen atoms bond one at a time instead of as pairs. In their model, there is never coexistence [see also Mountford and Sudbury (1992) for an extension of their results]. Durrett and Swindle (1994) modified the ZGB model by including fast stirring. That is, for each pair of neighboring sites, the values are exchanged at a rate that is fast compared to the adsorption rates. Their approach can be justified by the observation of Engel and Ertel (1979) that carbon monoxide molecules are highly mobile on the catalyst surface (oxygen atoms also have some mobility). In their model, all three phases (oxygen poisoning, coexistence, monomer poisoning) are present. No results regarding coexistence for the original ZGB model are currently known.

We take a different approach. We replace the oxygen molecule in the ZGB model by a large polymer consisting of $N^{2}$ identical atoms arranged in a square of length $N$, with $N$ quite large. Otherwise, the adsorption, disassoci- 


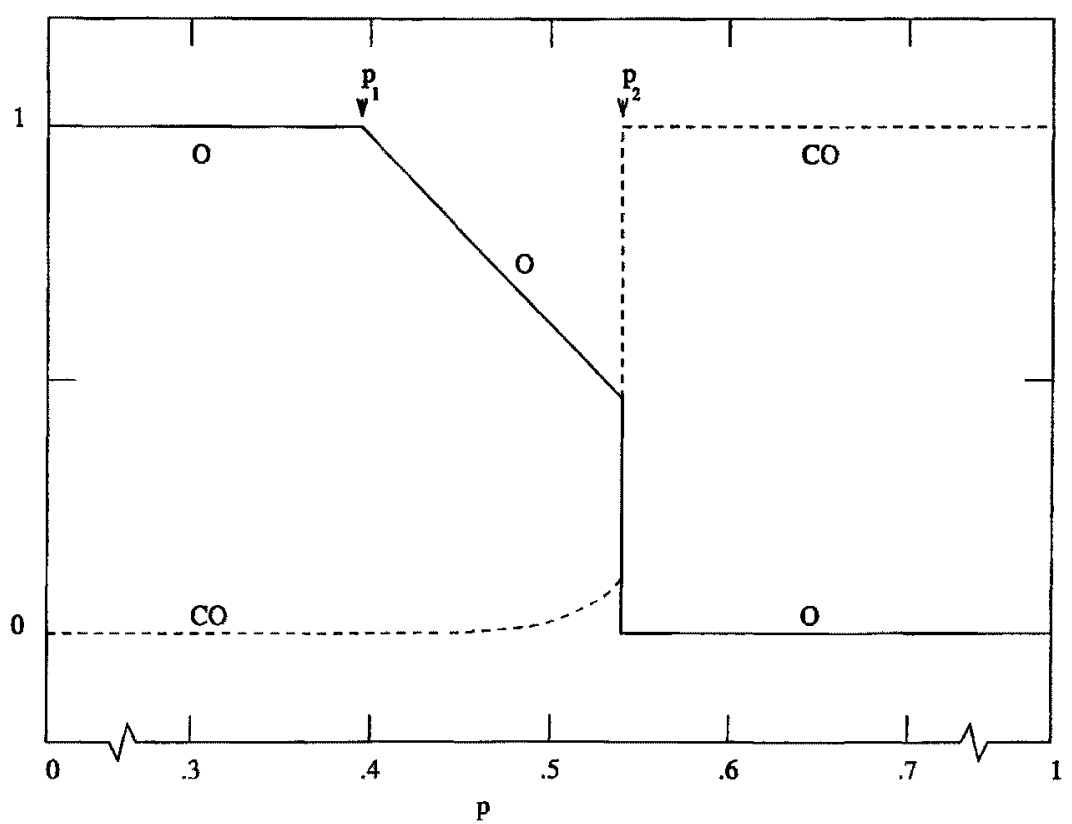

Fig. 1. The equilibrium density of $\mathrm{O}$ (solid lines) and $\mathrm{CO}$ (dashed lines) as functions of $p$. Transitions occur at $p_{1}$ and $p_{2}$.

ation, reaction and desorption proceed as before, although the allowed range of interaction remains to be specified. This modification results in the loss of one phase: polymer poisoning will not occur. Depending on the range of interaction, however, there will be situations in which there are two phases as the parameter $p$ is varied: one phase in which both components coexist, and another phase in which monomer poisoning occurs. For other ranges of interaction, monomer poisoning will always occur. Unlike previous rigorous results, the spatial geometry of configurations plays a major role here. The results presented here were previously announced in Bramson and Neuhauser (1992).

After these introductory comments, we are now ready to present the monomer-polymer model considered in this paper and state the accompanying results. The model is a continuous time Markov process in which the state $\xi_{t}$ at time $t$ is a function $\mathbf{Z}^{2} \rightarrow\{0,1,2\}$ (i.e., each site in $\mathbf{Z}^{2}$ is assigned the value 0,1 or 2 ). If $\xi_{t}(x)=0$, we say that $x$ is vacant; if $\xi_{t}(x)=1, x$ is occupied by a monomer, and if $\xi_{t}(x)=2, x$ is occupied by a polymer atom. The evolution of the process is given by the following rules.

(i) 0 's turn into 1's at rate 1, that is, monomers bond to vacant sites at rate 1.

(ii) $N \times N$ squares of 0's turn into $N \times N$ squares of 2's at rate $\lambda$, that is, $N \times N$ squares of vacant sites are occupied by polymers at rate $\lambda$. Polymers disassociate into single polymer atoms upon bonding, each occupying a single site. 
(iii) When a monomer bonds to a site $x$ as in (i) and there is a polymer atom at at least one site $y$ with $\|x-y\|_{\infty} \leq R$, then both $x$ and one such $y$ are immediately vacated; that is, the two atoms desorb. Similarly, when a polymer bonds to the lattice, the individual polymer atoms immediately interact with monomers within distance $R$, whenever possible.

Here, $\|x\|_{\infty} \leq R$ means $\max \left\{\left|x_{1}\right|,\left|x_{2}\right| \leq R\right.$, where $x=\left(x_{1}, x_{2}\right)$. The parameter $R, R \geq 1$, is the range of interaction; $N$ is the polymer length. "Polymer" always refers to an entire $N \times N$ block of polymer atoms, and "polymer atom" to a single component of a polymer.) The rates $\lambda \in[0, \infty)$ are allowed, with the probabilities $p$ chosen previously corresponding to $1 /(\lambda+1)$ in this setting. So that $\xi_{t}$ will be right continuous, $\xi_{t}(x)$ will denote the state at $x$ after desorption. [Without loss of generality, assume $\xi_{t}(x)$ to be continuous at $t=0$.]

Rules (i) and (ii) describe the adsorption mechanism, and rule (iii) describes the reaction between the components and the desorption of the product. One can use the standard tool of graphical representations to construct the monomer-polymer process in a more concrete setting. For each $x \in \mathbf{Z}^{2}$ let $\mathscr{U}_{n}^{x}$ and $\mathscr{V}_{n}^{x}, n=1,2, \ldots$, denote the successive times at which jumps occur (i.e., regeneration times) for independent Poisson processes with rates 1 and $\lambda$, respectively. We stipulate that at each time $\mathscr{U}_{n}^{x}$, a monomer bonds at $x$ provided $x$ is vacant, and that at each time $\mathscr{V}_{n}^{x}$, a polymer bonds to $x+[0, N)^{2}$ provided all sites in that set are vacant. These operations correspond to (i) and (ii). Desorption as in (iii) is specified by independent uniform-[0,1] random variables $\mathscr{W}_{s}^{x, y}, s \geq 0,\|x-y\|_{\infty} \leq R$, at those times $s$ when monomers and polymer atoms are within distance $R$. Prioritizing desorption for pairs $(x, y)$ with $\xi_{s}(x)=1$ and $\xi_{s}(y)=2$ by $\mathscr{W}_{s}^{x, y}$ then determines which sites become vacant. (Although we will restrict ourselves to this scheme, the precise rule used to match monomers and polymer atoms is not important). The random variables $\mathscr{U}_{n}^{x}, \mathscr{V}_{n}^{x}, \mathscr{W}_{s}^{x, y}$, with $n=1,2, \ldots, s>0$, $x, y \in \mathbf{Z}^{2}$, define the percolation substructure of $\xi_{t}$. The $\sigma$-algebra generated by these variables up to time $t$ and by $\xi_{0}$ will be denoted by $\mathscr{F}_{t}$.

We also need to introduce the following set of initial configurations. Let $\Xi_{1}$ denote the set of configurations which have infinitely many monomers or vacant sites. Also, let $\Xi_{2}$ denote those configurations which have infinitely many $N \times N$ squares not containing any monomers. Set $\Xi=\Xi_{1} \cap \Xi_{2}$. The condition $\Xi_{1}$ will rule out trivial cases of poisoning by the polymers, and $\Xi_{2}$, poisoning by the monomers.

As we see below, the behavior of the model depends on how large the range of interaction is relative to the polymer size. The following result shows that when the range of interaction is small relative to the polymer size, monomer poisoning occurs for all values of $\lambda$.

THEOREM 1.1. Assume that $1 \leq R \leq m_{0} N$ and that $\xi_{0} \in \Xi_{1}$. For appropriate $m_{0}>0$, monomer poisoning occurs for the process $\xi_{t}$ with probability 1 for all $\lambda \geq 0$. 
It is somewhat surprising that coexistence cannot occur in the above setting no matter how large $\lambda$ is; this behavior differs from simulations in the literature, which were done for small $N$.

The behavior is more complex when the range is large relative to the polymer size. On the one hand, monomer poisoning must always occur for small values of $\lambda$.

Theorem 1.2. Assume that $\xi_{0} \in \Xi_{1}$. For all $\lambda<1 / N^{2}$, monomer poisoning occurs for $\xi_{t}$ with probability 1.

On the other hand, if the range is large, coexistence occurs for large enough $\lambda$. By survival of monomers or polymer atoms, we mean that for $\varepsilon>0$ and large enough $L$, any given $L \times L$ square will possess that type of atom with probability at least $1-\varepsilon$ at all large enough times. Survival of both types is equivalent to coexistence. Theorem 1.3 gives sufficient conditions for the survival of polymer atoms, and Theorem 1.4 for survival of monomers.

THEOREM 1.3. Assume that $R=m N$ and that $\xi_{0} \in \Xi_{2}$. For $m \geq m_{1}$, appropriate $m_{1}$ and large enough $\lambda$ (not depending on $m, N$ ), polymer atoms survive for $\xi_{t}$.

THEOREM 1.4. Assume that $R=m N$, with $m \geq m_{1}$ and $N \geq N_{1}$, and that $\xi_{0} \in \Xi_{1}$. For appropriate $m_{1}$ and $N_{1}$ (and arbitrary $\lambda$ ), monomers survive for $\xi_{t}$.

Theorems 1.3 and 1.4 together show that coexistence occurs for large $m, N$ and $\lambda$, if $\xi_{0} \in \Xi$. We note that although Theorem 1.4 gives conditions for the survival of monomers, these monomers will, for large $m$ and $\lambda$, be very rare, with sites typically either being vacant or occupied by polymer atoms. (We comment on this in Section 5.)

By considering the Cesaro average of the distributions of $\xi_{s}, 0 \leq s \leq t$, and taking the limit over an appropriate subsequence, one obtains an equilibrium $\nu$. [This standard construction can be found in, e.g., Proposition 1.8 of Liggett (1985).] Theorems 1.3 and 1.4 imply that for sufficiently large $m, N$ and $\lambda$, such a $\nu$ possesses vacant sites, and hence an active reaction. Interesting questions in this context concern the uniqueness of $\nu$, and possible convergence results. On the other hand, in the cases given by Theorems 1.1 and 1.2, the limit is given by the state consisting only of monomers. Note that the above results do not rule out the possibility of polymer poisoning for intermediate values of $m$ (with large $N$ ) as specified in Theorem 1.3, although such behavior is quite unlikely. Most plausible is the following scenario for the system as one varies the parameters: for small $N$ (as in the ZGB model), poisoning by either type is possible, depending on the rate $\lambda$, and irrespective of $m$. Past some critical $N_{P}$ (presumably, depending on $m$, but bounded), polymer poisoning can no longer occur. (It seems likely, in fact, that the polymer poisoning in the ZGB model is lost for polymers of size at least three 
(of any shape), and even for dimers when $R>1$.) Also, past some critical $N_{M}$, monomer poisoning occurs for small $m$, irrespective of $\lambda$. As in the ZGB model, there are presumably always critical $\lambda_{1}$ and $\lambda_{2}$, with $0<\lambda_{1} \leq \lambda_{2} \leq \infty$ and depending on $N$ and $m$, which partition $\mathbf{R}^{+}$into monomer poisoning, coexistence and polymer poisoning intervals in the obvious order.

The monomer-polymer process we are considering can be formulated in $\mathbf{Z}^{d}, d \geq 1$, as well, although nothing basic changes in that setting. We also note that one can incorporate another parameter into our process by assuming the interaction between monomers and polymer atoms is not instantaneous, but occurs after a random time according to an exponential distribution with mean $\mu$. One can show that, for fixed $\mu>0$, monomer poisoning occurs for large enough $N$ irrespective of $m$ and $\lambda$. (Also, for any $N>1$, monomer poisoning occurs if $\mu$ is large.) For fixed $N$ and small enough $\mu$, the analogs of Theorems 1.1-1.4 hold as before.

The remainder of the paper is organized as follows. In Section 2, we demonstrate Theorem 1.1, and in Section 3, we demonstrate Theorem 1.2. For Theorems 1.3 and 1.4, we need to employ a rescaling argument that involves comparison of $\xi_{t}$ with oriented percolation. This procedure is reviewed in Section 4. Theorem 1.3 is then demonstrated in Section 5. Theorem 1.4 is considerably more difficult than the other three results, and its proof occupies the remaining three sections of the paper. In Section 6 , the theorem is demonstrated under the restriction that $\lambda \leq \delta m^{2}$, for appropriate $\delta>0$. The proof of the key result, Proposition 6.3, is deferred to Section 7. In Section 8, the case $\lambda>\delta m^{2}$ is handled.

2. Monomer poisoning for short range. We demonstrate Theorem 1.1 in this section. The theorem states that if the range of interaction $R$ is a small enough multiple of the polymer length $N$, then monomer poisoning must occur irrespective of the rate $\lambda$. The basic idea is that in order to have room to bond to the surface, a polymer requires a vacant $N \times N$ square. If there is no such square locally but nevertheless individual vacant sites, monomers will bond and react with nearby polymer atoms. In this manner, monomers are able to gradually displace polymer atoms without usually creating such $N \times N$ squares, and are thus able to ultimately poison the surface.

We first partition $\mathbf{R}^{2}$ into $\lfloor N / 2] \times[N / 2]$ squares and note that any $N \times N$ square must cover at least one of these smaller squares. ( $\lfloor x\rfloor$ denotes the integer part of $x$, and $\lceil x\rceil$, the smallest integer at least as large as $x$.) Fixing one $[N / 2] \times\lfloor N / 2]$ square $B$, we let $\mathscr{A}_{p}$ denote the set of configurations $\xi$ possessing at least $k_{0}$ polymer atoms in $B$, where $k_{0}$ will be chosen later. Also, denote by $\sigma$ the next (random) time at which a bonding polymer covers $B$, and $\tau$ the next time a bonding polymer and $B$ intersect so as to contain at least an $N / 9 \times N / 9$ square; in the latter case, we say that the polymer and $B$ overlap. Of course, $\sigma \geq \tau$, with both possibly being infinite.

The following proposition serves as our main tool here. It states that under $\xi_{0} \in \mathscr{A}_{p}$, the likelihood of the first overlapping polymer covering $B$ is small. 
Proposition 2.1. Assume that $1 \leq R \leq m_{0} N$ for appropriate $m_{0}>0$, and that $\xi_{0} \in \mathscr{A}_{p}$. Then,

$$
P(\sigma=\tau<\infty) \leq 2^{-1 /\left(720 m_{0}^{2}\right)} .
$$

Let $\sigma_{1}, \sigma_{2}, \ldots$ denote the successive times at which bonding polymers cover $B$, and $\tau_{1}, \tau_{2}, \ldots$ the times bonding polymers overlap $B$. Also, let $n_{\sigma}(t)$ and $n_{\tau}(t)$ denote the number of such events by time $t$. We denote by $B_{I}$ the $\lceil N / 6\rceil \times[N / 6\rceil$ square centered at the middle of $B$, and let $\mathscr{A}_{m}$ be those configurations $\xi$ possessing at least one monomer in $B_{I}$. Using Proposition 2.1, one can deduce the following bounds on $E\left[n_{\sigma}(t)\right]$.

Proposition 2.3. Assume that $1 \leq R \leq m_{0} N$ for appropriate $m_{0}>0$.

(a) For all $\xi_{0}$ and $t$,

$$
E\left[n_{\sigma}(t)\right] \leq 2^{-1 /\left(720 m_{0}^{2}\right)} E\left[n_{\tau}(t)\right]+2 .
$$

(b) If $\xi_{0} \in \mathscr{A}_{m}$, then

$$
E\left[n_{\sigma}(t)\right] \leq 2^{-1 /\left(720 m_{0}^{2}\right)} E\left[n_{\tau}(t)\right]
$$

for all $t$.

Before demonstrating Propositions 2.1 and 2.3, we see how Theorem 1.1 follows from (2.4) and (2.5). Each time a polymer overlaps a square $B$, it must cover either $B$ or one of the square's eight nearest neighbors in the $\|\cdot\|_{\infty}$ norm. So $E\left[n_{\tau}(t)\right]$ is at most the sum of the covering expectations for these nine squares. It follows that

$$
E\left[n_{\tau}(t)\right] \leq 9 E\left[n_{\sigma}^{1}(t)\right],
$$

where $n_{\sigma}^{1}(t)$ corresponds to the square $B_{1}$ among these nine squares possessing the highest such expectation. Combining (2.4) and (2.6) yields

$$
E\left[n_{\sigma}(t)\right] \leq \frac{1}{2} E\left[n_{\sigma}^{1}(t)\right]+2
$$

for small enough $m_{0}$, and hence, that

$$
E\left[n_{\sigma}^{1}(t)\right]-4 \geq 2\left(E\left[n_{\sigma}(t)\right]-4\right) .
$$

One can iterate this procedure, successively choosing neighboring squares $B_{2}, B_{3}, \ldots$ satisfying the analog of (2.6). The covering expectation of the $i$ th such square thus satisfies

$$
E\left[n_{\sigma}^{i}(t)\right]-4 \geq 2^{i}\left(E\left[n_{\sigma}(t)\right]-4\right) .
$$

It is clear from (2.8) that for any $\lfloor N / 2\rfloor \times\lfloor N / 2\rfloor$ square $B$ and any $\xi_{0}$,

$$
E\left[n_{\sigma}(t)\right] \leq 4 \text { for all } t \text {. }
$$

Otherwise, $\lim _{i \rightarrow \infty} E\left[n_{\sigma}^{i}(t)\right]=\infty$, but since polymers bond at only the fixed rate $\lambda$, this is not possible. So, the number of polymers ever covering a square $B$ is almost surely finite. A little thought shows that this implies poisoning for the system. Such poisoning occurs independently of the initial configuration. 
For $\xi_{0} \in \Xi_{1}$, this poisoning must be by monomers. To show this, first note that under $\Xi_{1}$, for given $i \in \mathbf{Z}^{+}$, there is with probability 1 an $[N / 2] \times[N / 2]$ square $B$ such that at $t=1$, all squares $B^{\prime}$ within distance $i$ of $B$ possess at least one monomer in $B_{I}^{\prime}$. One can repeat the reasoning used in (2.7) for the process $\hat{\xi}_{t}={ }_{\text {def }} \xi_{t+1}$, but with (2.5) substituted for (2.4), since $\hat{\xi}_{0} \in \mathscr{A}_{m}$. This gives

$$
E\left[\hat{n}_{\sigma}(t)\right] \geq \frac{1}{2} E\left[\hat{n}_{\sigma}^{1}(t)\right]
$$

for all $t$, where $\hat{n}_{\sigma}(t)$ and $\hat{n}_{\sigma}^{1}(t)$ count the covering times corresponding to those in (2.7). One can iterate (2.10) $i$ times to obtain

$$
E\left[\hat{n}_{\sigma}(t)\right] \leq 2^{-i} E\left[\hat{n}_{\sigma}^{i}(t)\right] .
$$

Inequalities (2.9) and (2.11) imply that for the above square $B$,

$$
P\left(\hat{n}_{\sigma}(t)>0\right) \leq E\left[\hat{n}_{\sigma}(t)\right] \leq 2^{2-i} .
$$

The analog of (2.12) at each square neighboring $B$ likewise holds, but with the upper bound $2^{2-(i-1)}=2^{3-i}$ instead. So, a square $B$ can be chosen so that the probability it or any of its neighbors is covered by a polymer after $t=1$ for the process $\xi_{t}$ is as small as desired. But, in order for $B$ to be completely occupied by polymer atoms at some $t>1$, some bonding polymer must intersect with $B$, and this polymer must cover either $B$ or one of its neighbors. So, the probability that $B$ is completely occupied by polymer atoms at any $t>1$ can also be made as small as desired. Consequently, polymer poisoning occurs with zero probability. The only other possibility is poisoning by monomers, which implies Theorem 1.1.

Propositions 2.1 and 2.3 remain to be proved. We say that a bonding monomer is noninstantaneous if it does not immediately react with a polymer atom and desorb. The basic idea behind the proof of Proposition 2.1 is to show that for an initial configuration with a substantial number of polymer atoms in $B$, a noninstantaneous monomer will typically bond to $B_{I}$ before all of these atoms desorb. The presence of such a monomer will prevent the next overlapping polymer from covering $B$, and hence imply that $\sigma>\tau$. For this, we set $k_{0}=\left\lceil 1 / 720 m_{0}^{2}\right\rceil$, with $k_{0}$ being the number of polymer atoms in the definition of $\mathscr{A}_{p}$.

Proof of Proposition 2.1. Any site within $B_{I}$ is at least distance $N / 6-1$ from the boundary of $B$. Once a noninstantaneous monomer bonds to $B_{I}$, the only way for it to desorb is for a polymer to bond within distance $m_{0} N$ of the site. For small $m_{0}$, such a polymer contains sites at least distance $N / 9$ from the boundary of $B$, and hence overlaps $B$. Since $B$ is not vacant, this polymer does not cover $B$. It follows that once a noninstantaneous monomer bonds to $B_{I}, \sigma=\tau<\infty$ cannot occur.

No polymer can cover $B$ until all of the polymer atoms in $B$ at $t=0$ have desorbed. Since $\xi_{0} \in \mathscr{A}_{p}$, we know that there are at least $k_{0}$ such atoms. Wait until there are exactly $k_{0}$ such atoms left. If a noninstantaneous monomer bonds to $B_{I}$ before all of these $k_{0}$ atoms desorb, the situation reduces to that 
discussed in the previous paragraph. Also, if a polymer bonds within distance $R$ of $B_{I}$ over this period, then the polymer overlaps $B$, and so $\sigma>\tau$. To bound $P(\sigma=\tau<\infty)$, it thus suffices to obtain an upper bound on the probability of the $k_{0}$ atoms desorbing before either of these two events.

To react with a given polymer atom, a monomer must bond within distance $R$. There are at most $(2 R+1)^{2}$ such sites. The number of vacant sites within distance $R$ of any of the $k_{0}$ polymer atoms is therefore at most

$$
k_{0}(2 R+1)^{2} \leq 9 k_{0} m_{0}^{2} N^{2} \leq \frac{N^{2}}{72},
$$

for $m_{0}$ small. Since $\left|B_{I}\right| \geq N^{2} / 36, B_{I}$ contains at least $N^{2} / 72$ sites not within $R$ of any of these polymer atoms. Also, there are no other polymer atoms within distance $R$ of $B_{I}$. The probability of any of the $k_{0}$ polymer atoms desorbing before time $\tau$ and before a noninstantaneous monomer bonds to $B_{I}$ is therefore at most $1 / 2$. One can repeat this reasoning $k_{0}$ times to conclude that the probability of all the polymer atoms desorbing before either of these two events is at most $1 / 2^{k_{0}}$. Substituting in for $k_{0}$, one gets (2.2).

The intersection of an overlapping, bonding polymer with $B$ contains at least an $N / 9 \times N / 9$ square. For $m_{0} \leq 1 / 18$, there is an $N / 18 \times N / 18$ subsquare of polymer atoms which are too far inside the interior of this square to immediately react with any monomers. Under $N \geq 1 / m_{0}$ and $k_{0}=\left[1 / 720 m_{0}^{2}\right\rceil$, it automatically follows that such a configuration possesses at least $k_{0}$ polymer atoms in $B$, and hence is in $\mathscr{A}_{p}$. One is therefore able to repeatedly apply Proposition 2.1 to $\xi_{t}$ at the times $\tau_{1}, \tau_{2}, \ldots$ to conclude that the probability of an overlapping polymer covering $B$ is at any step small. Together with a martingale argument, this will imply Proposition 2.3. For the martingale, we employ the $\sigma$-algebras $\mathscr{G}_{l}=\mathscr{F}_{r_{l}}, l=1,2, \ldots$

Proof of Proposition 2.3. As shown above, any polymer which overlaps $B$ contains at least $k_{0}$ atoms which do not immediately desorb. So, irrespective of $\xi_{0}$, if $\tau_{1}<\infty$, then $\xi_{\tau_{1}} \in \mathscr{A}_{p}$, with the corresponding polymer possibly covering $B$. Employing this observation and the strong Markov property, one can restart the process at time $\tau_{1}$. The bound (2.4) then follows from

$$
E\left[n_{\sigma}(t)\right] \leq 2^{-1 /\left(720 m_{0}^{2}\right)} E\left[n_{\tau}(t)+1\right] \text { for } \xi_{0} \in \mathscr{A}_{p} .
$$

To demonstrate (2.14), we introduce the process $Z_{l}, l=0,1, \ldots$, with $Z_{0}=0$ and $Z_{l}=Y_{1}+\cdots+Y_{l}$. We set $Y_{i}=2^{-1 /\left(720 m_{0}^{2}\right)}$ if the bonding polymer which overlaps $B$ at time $\tau_{i}<\infty$ also covers $B$, and $Y_{i}=-1$ if either the polymer does not cover $B$ or $\tau_{i}=\infty$. (If $\tau_{l}=\infty$, set $Z_{l}=Z_{l+1}=\cdots$.) It follows from Proposition 2.1, that for $\xi_{0} \in \mathscr{A}_{p}$,

$$
E\left[Y_{i} \mid \mathscr{G}_{i-1}\right] \leq 0 \text { for all } i \geq 1 \text {, }
$$

and so $\left\{Z_{l}\right\}$ is a supermartingale with respect to $\left\{\mathscr{G}_{l}\right\}$. Let

$$
\rho_{t}=\min \left\{l: \tau_{l} \geq t\right\} \text {. }
$$


Note that $\rho$ is essentially the inverse of $\tau$, with

$$
\tau_{\rho_{t}-1}<t \leq \tau_{\rho_{t}}
$$

For given $t, \rho_{t}$ is a stopping time with respect to $\left\{\mathscr{G}_{l}\right\}$. Since the number of polymers which overlap $B$ by time $t$ is bounded by a Poisson random variable with mean $(9 / 4) \lambda N^{2} t, E\left[\rho_{t}\right]<\infty$. Note that the increments $Y_{i}$ of $Z_{l}$ are bounded. One can therefore apply a standard version of the optional sampling theorem to conclude that, for any $t \geq 0$,

$$
E\left[Z_{\rho_{t}}\right] \leq E\left[Z_{0}\right]=0 .
$$

It follows from (2.17) and the definition of $n_{\sigma}(t)$ and $n_{\tau}(t)$, that for $\xi_{0} \in \mathscr{A}_{p}$,

$$
E\left[n_{\sigma}\left(\tau_{\rho_{t}}\right)\right] \leq 2^{-1 /\left(720 m_{0}^{2}\right)} E\left[n_{\tau}\left(\tau_{\rho_{t}}\right)\right] .
$$

[If $\tau_{\rho_{t}}=\infty$, interpret $n_{\tau}\left(\tau_{\rho_{f}}\right)$ to include an "overlapping polymer at $t=\infty$ " because of the definition of $Y_{i}$ below (2.14).] By (2.16),

$$
n_{\sigma}(t) \leq n_{\sigma}\left(\tau_{\rho_{t}}\right) \text {. }
$$

Similarly,

$$
n_{\tau}\left(\tau_{\rho_{t}}\right)=n_{\tau}\left(\tau_{\rho_{t}-1}\right)+1 \leq n_{\tau}(t)+1
$$

Substituting these bounds into (2.18) yields (2.14), which in turn implies (2.4).

The bound (2.5) follows in place of (2.14) under the condition $\xi_{0} \in \mathscr{A}_{m}$. To see this, note that for a monomer in $B_{I}$ to desorb, an overlapping polymer must bond. Since $B$ is not vacant, this polymer does not cover $B$. In particular, the overlapping polymer at $\tau_{1}$ does not cover $B$. This means that $Y_{1}=-1$, where $Y_{i}$ is defined as above. Continuing as before, one obtains the analog of (2.18), but with $n_{\tau}\left(\tau_{\rho_{\mathrm{t}}}\right)$ replaced by $n_{\tau}\left(\tau_{\rho_{t}}\right)-1$. Using (2.19) and (2.20) as before, one gets (2.5).

3. Monomer poisoning for small $\lambda$. Here, we demonstrate Theorem 1.2 , namely, that monomer poisoning must occur for the monomer-polymer process $\xi_{t}$ if $\lambda<1 / N^{2}$, irrespective of the polymer length $N$ or the range $R$. Such behavior is, to be sure, not surprising. Even under "ideal" circumstances for the polymer, when all sites are vacant, monomers bond at a higher rate than do polymer atoms; the presence of monomers and polymer atoms on the surface just imbalances these rates further. One can therefore think in terms of random walks with negative drift, and use a modification of the accompanying exponential supermartingales to analyze the process.

For any configuration $\xi$ of vacant sites, monomers and polymer atoms, let $\hat{\xi}$ denote the reassignment of values, with $\hat{\xi}(x)=-1$ for a monomer at $x$, $\hat{\xi}(x)=0$ when vacant, and $\hat{\xi}(x)=1$ for a polymer atom. Since the number of sites on the lattice is infinite, the counter

$$
Y_{\alpha}(\xi)=\sum_{x \in \mathbf{Z}^{2}} \exp \left(-\alpha\|x\|_{\infty}\right) \hat{\xi}(x), \quad \alpha>0,
$$


will be useful. Note that $Y_{\alpha}(\xi)$ is bounded for given $\alpha$, and that if $\xi^{2}(x)=2$ for all $x \in \mathbf{Z}^{2}$, one has

$$
h_{\alpha}={ }_{\text {def }} Y_{\alpha}\left(\xi^{2}\right)=\sum_{x \in Z^{2}} \exp \left(-\alpha\|x\|_{\infty}\right) .
$$

Our main tool is the function of $\xi_{t}$,

$$
Z_{\alpha, \beta}(t)=\exp \left(\beta Y_{\alpha}\left(\xi_{t}\right)\right), \quad \alpha, \beta>0 .
$$

Lemma 3.4. For small enough $\alpha$ and $\beta, Z_{\alpha, \beta}(t)$ is a supermartingale.

Proof. The function $Z_{\alpha, \beta}(t)$ changes when either a monomer bonds at some vacant site $x$, or a polymer bonds to the vacant sites $x+[0, N)^{2}$; the resulting atoms will then react with atoms of the opposite type within distance $R$. When such a bonding has occurred at $x$, the probability is at least $1 /(\lambda+1)$ that it was a monomer which bonded. In this case, $Y_{\alpha}$ decreases by at least $\exp \left[-\alpha\left(\|x\|_{\infty}+R\right)\right]$. When a polymer bonds, $Y_{\alpha}$ increases by at most $N^{2} \exp \left[-\alpha\left(\|x\|_{\infty}-N-R\right)\right]$. The expected change in $Z_{\alpha, \beta}$ following bonding at $x$ at time $t$ is therefore at most

$$
\begin{aligned}
& \left(\frac{1}{\lambda+1} \exp \left[-\beta \exp \left(-\alpha\left(\|x\|_{\infty}+R\right)\right)\right]\right. \\
& \left.+\frac{\lambda}{\lambda+1} \exp \left[\beta N^{2} \exp \left(-\alpha\left(\|x\|_{\infty}-N-R\right)\right)\right]-1\right) Z_{\alpha, \beta}(t-) .
\end{aligned}
$$

Assume that $\alpha \leq 1$ and $\beta$ is small. Expansion of the coefficient of $Z_{\alpha, \beta}(t-)$ shows that it is at most

$$
\begin{aligned}
& \frac{\beta \exp \left(-\alpha\left(\|x\|_{\infty}+R\right)\right)}{\lambda+1}\left(\lambda N^{2} \exp (\alpha(N+2 R))-1\right) \\
& +C_{N, R} \beta^{2} \exp \left(-2 \alpha\|x\|_{\infty}\right),
\end{aligned}
$$

for some constant $C_{N, R}$. For small enough $\alpha$ and $\beta$, this is negative because $\lambda<1 / N^{2}$. Since this is true irrespective of the state $\xi_{t-}$, it follows that $Z_{\alpha, \beta}(t)$ is a supermartingale.

Fix $\alpha$ and $\beta$ small enough so that Lemma 3.4 holds. Since $Z_{\alpha, \beta}(t)$ is a bounded supermartingale, it follows from basic theory that $Z_{\alpha, \beta}(\infty)=$ $\lim _{t \rightarrow \infty} Z_{\alpha, \beta}(t)$ exists almost surely, and that

$$
E\left[Z_{\alpha, \beta}(\infty)\right] \leq Z_{\alpha, \beta}(0) \text {. }
$$

The existence of the limit implies that poisoning must occur almost surely for $\xi_{t}$. Together with (3.2) and the positivity of $Z_{\alpha, \beta},(3.5)$ implies that

$$
P \text { (polymer poisoning occurs) } \leq Z_{\alpha, \beta}(0) \exp \left(-\beta h_{\alpha}\right) \text {. }
$$

Recall that $\Xi_{1}$ denotes those states with infinitely many monomers or vacant sites. It is easy to check, for $\xi_{0} \in \Xi_{1}$, that

$$
h_{\alpha}-Y_{\alpha}\left(\xi_{0}\right) \rightarrow \infty \text { as } \alpha \rightarrow 0 \text {. }
$$


So, for fixed $\beta$ and $\alpha \rightarrow 0$, the right side in (3.6) goes to 0 , if $\xi_{0} \in \Xi_{1}$. Consequently, polymer poisoning never occurs, and in fact,

$$
P \text { (monomer poisoning occurs })=1 \text {. }
$$

This implies Theorem 1.2. [Mountford and Sudbury (1992) use a related argument for the corresponding monomer-monomer process. Instead of the exponential supermartingale in (3.3), they employ the analog of $Y_{\alpha}$, together with its translations.]

4. Comparison with oriented percolation. For Theorems 1.3 and 1.4, we will employ a rescaling argument. It is by now a standard technique and has been applied frequently. [See, e.g., Bramson (1989), Bramson and Durrett (1988), Bramson and Neuhauser (1993), Durrett (1992) and Durrett and Neuhauser (1994); the argument is reviewed in Durrett (1991).] The basic idea is to show that, for given $\delta>0$, members of the family of processes under consideration, when viewed on suitable length and time scales, dominate an oriented site percolation process in which sites are open with probability $1-\delta$. (The sites may be $j$-dependent.) Since oriented site percolation percolates for $\delta$ close enough to 0 , this will then imply that these processes survive, and have the desired properties.

We review the above procedure. Oriented site percolation can be defined as follows. For $z=\left(z_{1}, z_{2}\right) \in \mathbf{Z}^{2}$, let $\mathscr{L}=\left\{(z, k) \in \mathbf{Z}^{2} \times \mathbf{Z}: z_{1}+z_{2}+k\right.$ is even $\}$. The oriented site percolation process is a collection of random variables $\{\sigma(z, k):(z, k) \in \mathscr{L}\}$, with values in $\{0,1\}$, that indicate whether the sites in $\mathscr{L}$ are open (1) or closed (0). We say that the process is $j$-dependent with density at least $1-\delta$ if for any sequence $\left(z_{a}, k_{a}\right), a=1,2, \ldots, h$, satisfying $\left\|z_{a_{1}}-z_{a_{2}}\right\|_{1}>2 j$ whenever both $a_{1} \neq a_{2}$ and $k_{a_{1}}=k_{a_{2}}$, then

$$
P\left(\sigma\left(z_{a}, k_{a}\right)=0 \text { for } a=1, \ldots, h\right) \leq \delta^{h} .
$$

By an open path from $(y, 0)$ to $(z, k), k \geq 1$, we mean a sequence of points $\zeta_{0}=(y, 0), \zeta_{1}, \zeta_{2}, \ldots, \zeta_{k}=(z, k)$ in $\mathscr{L}$, such that for $l=1, \ldots, k, \zeta_{l}=\zeta_{l-1}+$ $\left(e_{i}, 1\right)$ or $\zeta_{l}=\zeta_{l-1}+\left(-e_{i}, 1\right)$ for some $i \in\{1,2\}$, where $e_{i}$ is the $i$ th unit vector and all the $\zeta_{l}$ 's, $l=1, \ldots, k$, are open.

Let $A \subset \mathbf{Z}^{2}$ and define

$$
\begin{array}{r}
W_{k}^{A}=\{z: \text { there is an open path from }(y, 0) \\
\text { to }(z, k) \text { for some } y \in A\} .
\end{array}
$$

We think of $W_{k}^{A}$ ( $W_{k}$ for short) as the set of wet sites at time $k$ connected to the source $A$ at time 0 . Also, set

$$
\Omega_{\infty}^{\{y\}}=\bigcap_{k=0}^{\infty}\left\{W_{k}^{\{y\}} \neq \varnothing\right\},
$$

with $\Omega_{\infty}^{A}=\cup_{y \in A} \Omega_{\infty}^{\{y\}}$. When $\omega \in \Omega_{\infty}^{A}$, that is, there is an infinite open path starting at $A \times\{0\}$, we say percolation occurs from $A$. The following is, for $j$-dependent percolation, a slight variant of results on 1-dependent percolation given on pages 204 and 205 of Durrett and Neuhauser (1991). [A detailed 
exposition on independent oriented site percolation can be found in Durrett (1984). In Liggett, Schonmann and Stacey (1995), it is shown that $j$-dependent percolation can be coupled with independent percolation so as to dominate the latter and so that the exceptional probabilities $\delta$ converge to 0 in both cases; this allows one to automatically extend various percolation results to the $j$-dependent setting.] We denote by $B_{L^{\prime}}^{z}$ the square of length $L^{\prime}, L^{\prime}>0$, centered at $z \in \mathbf{Z}^{2}$.

LEMMA 4.4. Let $\{\sigma(z, k):(z, k) \in \mathscr{L}\}$ be a j-dependent oriented site percolation process with density at least $1-\delta$, for $\delta>0$. If $\delta$ is sufficiently small, then $P\left(\Omega_{\infty}^{\{0\}}\right)>0$. Fix such a $\delta$. Then, there exists $\gamma>0$ so that for any $\varepsilon>0$ and large enough $L^{\prime}$,

$$
\limsup _{k \rightarrow \infty} \max _{|z|<\gamma k} P\left(W_{k}^{\{0\}} \cap B_{L^{\prime}}^{z}=\varnothing ; \Omega_{\infty}^{\{0\}}\right)<\varepsilon .
$$

That is, on the set $\Omega_{x}^{\{0\}}$, a large square centered inside the cone $|z|<\gamma k$ will at large times $k$ typically contain at least one wet site. The relevant observation here is the same as that in Durrett and Neuhauser (1991), namely that, inside the cone, $W_{k}^{\{0\}}$ is at large $k$ typically equal to $W_{k}^{\mathbf{Z}^{2}}$.

The sources $A$ we will be employing will typically satisfy $|A|=\infty$. By choosing an infinite subset of $A$ whose elements are sufficiently separated from one another, one can construct an arbitrary number of copies of $W_{k}^{\{0\}}$ (up to translation) which are nearly independent of one another up to large times. Arguing in this manner, it is not difficult to show that $P\left(\Omega_{\infty}^{A}\right)=1$ for $|A|=\infty$. Applying this in conjunction with Lemma 4.4, it is also not difficult to show that for $|A|=\infty$,

$$
\limsup _{k \rightarrow \infty} \max _{|z|<\gamma k} P\left(W_{k}^{A} \cap B_{L^{\prime}}^{z} \neq \varnothing\right)<\varepsilon,
$$

where $\delta, \gamma, \varepsilon$, and $L^{\prime}$ are quantified as above. That is, large squares inside $|z|<\gamma k$ will at large times typically contain wet sites.

To compare our processes with oriented site percolation, we proceed as follows. Set

$$
\begin{aligned}
& \phi(z)=\left(M z_{1}, M z_{2}\right), \\
& B(z)=\phi(z)+B,
\end{aligned}
$$

for $z=\left(z_{1}, z_{2}\right) \in \mathbf{Z}^{2}$, where $B=(-M / 2, M / 2]^{2}, M \in 2 \mathbf{Z}^{+}$. The $M \times M$ squares $B(z)$ are centered at $\phi(z)$ and partition $\mathbf{Z}^{2}$. We will employ certain "good events" in the squares $B(z)$. The definition will depend on the precise situation, whether a sufficient number of monomers, polymer atoms, or vacant sites are present in $B(z)$. If the good event occurs in $B(z)$ at time $t$, with, for instance, $t=k T, T>0$, we then say $B(z)$ is occupied at this time. The terms $M$ and $T$ here are to be regarded as large and fixed. (In Sections $5-8$, we will set $M=2\lfloor R / 4\rfloor \approx R / 2$; the choice of $T$ will depend on the context.) 
Let $H_{k}(z)$ denote the event that $B(z)$ is occupied at time $k T$. The main step in the comparison with oriented site percolation is to show, for some fixed $j$, that the following bound holds for appropriately small $\delta$ : for all $z_{a}$, $a=1,2, \ldots, h$ satisfying $\left\|z_{a_{1}}-z_{a_{2}}\right\|_{1}>2 j, a_{1} \neq a_{2}$,

$$
P\left(\bigcap_{a=1}^{h} H_{k+1}^{c}\left(z_{a}\right) \mid \mathscr{F}_{k T}\right) \leq \delta^{h}
$$

whenever $\omega \in H_{k}\left(z_{a}^{\prime}\right)$ for some choice of $z_{a}^{\prime}$ with $\left\|z_{a}-z_{a}^{\prime}\right\|_{1}=1$ for all $a$. In the case $h=1,(4.8)$ states that $B(z)$ is not occupied at time $(k+1) T$ with probability at most $\delta$ if $B\left(z^{\prime}\right)$ is occupied at time $k T$ for some immediate neighbor $z^{\prime}$ of $z$. For $k \geq 0$, set $\sigma(z, k+1)=0$ if $B(z)$ is not occupied under these circumstances; otherwise, set $\sigma(z, k+1)=1$. Then, (4.1) is satisfied. Letting

$$
V_{k}=\left\{z \in \mathbf{Z}^{2}: B(z) \text { is occupied at time } k T\right\},
$$

and $W_{0} \subset V_{0}$, one can check by induction that

$$
W_{k} \subset V_{k}
$$

for all $k \geq 0$, where $W_{k}$ is defined as above. Under $\left|V_{0}\right|=\infty$, one can then apply (4.6) to $V_{k}$ to conclude that inside $|z|<\gamma k M$, each large $L^{\prime} \times L^{\prime}$ block of $M \times M$ squares typically contains at least one such occupied square at time $k T$. With a little more work, this will, in each case, imply that each $L \times L$ square, $L=L^{\prime} M$, will, with high probability, contain a monomer, polymer atom, or vacant site (depending on the result to be shown) at large enough times, and hence that the appropriate atom type survives.

Since the evolution of $\xi_{t}$ is time homogeneous, it will suffice to demonstrate (4.8) for $k=0$. It is also enough to show the inequality for $h=1$, if one can control the interaction between distinct blocks of sites. For attractive particle systems, this is often done by comparing the system to one with fixed configurations on certain boundary regions which separate these blocks [see, e.g., Bezuidenhout and Grimmett (1990), Bramson, Durrett and Swindle (1989), Durrett and Schinazi (1993) and Durrett and Swindle (1991, 1994)]. By choosing the "worst configuration" on the boundary, one obtains a new system which is pathwise dominated by the original system. If the boundary regions are specified to have width at least the range of interaction of the system, the evolution of the new system will be independent over the different blocks. Demonstration of (4.8) for $h=1$ for the new system will then imply analogous bounds for general $h$ for the original system.

The system $\xi_{t}$ is not attractive, however, and this increases the technical details in demonstrating (4.8). One can, nevertheless, follow the above outline for attractive processes, with some modification. (This procedure should work in general for nonattractive particle systems with finite range, when a rescaling argument can be applied.) Choosing $j=4$ in Section 5 and $j=5$ in Sections 6-8 in (4.8), we will specify boundary regions with width $R$, which separate the squares $\hat{B}\left(z_{a}\right)$ that contain $B\left(z_{a}\right)$. Once the configuration is 
chosen on these boundary regions, the evolution on the squares $\hat{B}\left(z_{a}\right)$ will be mutually independent. Since there is no uniformly worst configuration on the boundary for all paths, we take, for each path, a configuration (which will be allowed to change over time), which maximizes $\mathbf{1}\left(H_{1}^{c}\left(z_{a}\right)\right)$. Of course, the ensuing estimates then need to include the effect of varying the data on the boundary.

The main observation behind this reasoning is that one can simultaneously couple the different new systems, obtained by choosing the boundary data in all possible ways, so that the analog of $H_{1}^{c}\left(z_{a}\right)$ is in each case restricted to the same small set of paths. This coupling can be done by appropriately reordering, according to $x$, the times $\left\{\mathscr{U}_{n}^{x}\right\}$ and $\left\{\mathscr{V}_{n}^{x}\right\}$, given in Section 1 , at which monomers and polymers attempt to bond to $\mathbf{Z}^{2}$. Although the reordered times will still be given by independent Poisson processes, the choice of coupling for the positions $x \in \mathbf{Z}^{2}$ will frequently depend on the configurations of $\xi_{t}$ at those times. In practice, we will always be deriving bounds for functionals of $\xi_{t}$ [typically, Poisson-like processes, or in (7.14), a supermartingale], which will be increased or decreased by "favorable" or "unfavorable" monomer/polymer bonding and desorption. The favorable and unfavorable bonding and desorption can always be coupled in a natural way so as to provide uniform bounds for the desired functionals over the different systems. (For instance, in the proof of Lemma 5.6, we will require lower bounds on the number of polymers that bond in $B$. Under certain conditions, there will be at least $M^{2} / 3$ unoccupied positions in $B$ where polymers can bond. Their location will depend on the boundary data, which affects the matching of sites $x$ for the coupling of $\left\{\mathscr{V}_{n}^{x}\right\}$, but not the probabilities that are involved.) Together, these bounds on the desired functionals will imply the analog of (4.8).

Rather than complicate the notation in Sections 5-8 to include this coupling, we restrict ourselves to the case $h=1$ in the actual proofs, while pointing out briefly there how the coupling enters. (The reader will probably agree that the arguments in Sections 6-8 are already sufficiently cumbersome. The argument in Section 5 is not so long.) For those interested in tracking things down, we group together here the different instances in which such coupling is used. The same upper bounds on the number of monomers which attempt to bond, on the set of sites within distance $R$ of $B$, are employed in Lemmas 5.5, 7.17, 7.25, 8.17, 8.29 and 8.50, and in Proposition 6.16. These bounds allow one to control the number of polymer atoms which desorb from $B$ over that time period. The coupling here is the obvious "identity" coupling; in other cases, it depends on the configuration. Lower bounds on the number of monomers which bond and interact with polymer atoms in $B$ are used in Proposition 6.16 and Lemma 7.28; upper and lower bounds on the bonding rates are used in Lemma 7.14. Upper bounds on the number of polymers that bond over certain regions in $B$ are used in Lemmas 6.14 and 8.19, and lower bounds are used in Lemmas 5.6 and 8.50. The bounds in all the cases are based on the simple large deviations bounds for Poisson random variables given in (5.10). 
5. Polymer survival for large $\lambda$. It was shown in Sections 2 and 3 that either a short range of interaction or a low bonding rate for polymers ensures poisoning by monomers. Here, we demonstrate Theorem 1.3, which states that for large $m$ and $\lambda$, polymer atoms survive. The basic idea is that, on account of the long range, polymers will have plenty of room to bond in the active region between competing monomer and polymer clusters. Together with the choice of $\lambda$, this leads to the spread of such polymer clusters at the expense of monomers and hence to the survival of polymer atoms.

To demonstrate Theorem 1.3, we employ the framework provided in the previous section. We partition $\mathbf{Z}^{2}$ by $M \times M$ squares $B(z)$ as specified in (4.7), with $B=B(0)=(-M / 2, M / 2\rfloor^{2}$, and set $M=2\lfloor R / 4\rfloor$. All sites in neighboring squares are within distance $R$ of one another, with $M \approx R / 2$ since $R$ is large. Also, let $T=1 / 2,000 N^{2}$, where $T$ is the time increment used in the rescaling. We will say that $B(z)$ is occupied when there are at least $m^{2} / 20$ polymer atoms in $B(z)$, where, as always, $R=m N$.

The main goal of this section is to show the following result.

PROPOSITION 5.1. For any $\delta>0$, and sufficiently large $\lambda$ and $m$ (with $m$ depending on $\delta$, but neither $\lambda$ nor $m$ depending on $N$ ),

$$
P(B(0) \text { is not occupied at some } t \in[T, 2 T]) \leq \delta
$$

if $B(z)$ is occupied at time 0 for some $z$ with $\|z\|_{1}=1$.

As in Section 4, we let $H_{k}(z)$ denote the event that $B(z)$ is occupied at time $k T$. Since the evolution for $\xi_{t}$ is translation invariant in both space and time, inequality (5.2) implies, in particular, that

$$
P\left(H_{k+1}^{c}(z) \mid \mathscr{F}_{k T}\right) \leq \delta
$$

under $H_{k}\left(z^{\prime}\right)$ for any $z^{\prime}$ with $\left\|z-z^{\prime}\right\|_{1}=1$.

As discussed at the end of Section 4, we in fact require the multidimensional version given in (4.8) to show survival of polymer atoms. We find it convenient to set

$$
\hat{B}(z)=B_{M+2 R}^{z}
$$

here, which is the set of all points within distance $R$ of $B(z)$. We will need to be able to provide a lower bound on the number of polymers that bond in $B(0)$, and an upper bound on the number of monomers that bond in $\hat{B}(0)$ in order to derive (5.3), and, more generally, (4.8). Including a boundary region of width $R$ around $\hat{B}(0)$, to separate it from other squares $\hat{B}(z)$, gives a square of length $M+4 R \leq 10 M$ for large $R$. So, we stipulate that $\| z_{a_{1}}-$ $z_{a_{2}} \|_{1} \geq 10$ in (4.8). Reasoning as in the last three paragraphs of Section 4 will give the independence of the different squares needed for (4.8) to hold. (More detail is given at the end of this current section.)

Given (4.8), one can then compare the process $V_{k}$ in (4.9) with the corresponding oriented percolation $W_{k}$ by (4.10). This enables us to employ the bound (4.6). The assumption that $\xi_{0} \in \Xi_{2}$ in Theorem 1.3 implies that at 
any $t_{1}>0$, the number of occupied squares $B(z)$ is infinite with probability 1. Restarting the process then, and applying (4.6) and (4.10), it follows that for large enough $L^{\prime}$, and for $\delta$ chosen small enough in (5.3),

$$
\limsup _{k \rightarrow \infty} \max _{|\boldsymbol{z}|<\gamma k} P\left(V_{k} \cap B_{L^{\prime}}^{z}=\varnothing\right)<\varepsilon
$$

for fixed $\varepsilon>0$. This implies that the polymer atoms in $\xi_{t}$ survive at the times $k T, k=0,1,2, \ldots$. Employing (5.2) over the times $(T, 2 T)$ for the interval $(k T,(k+1) T)$, it follows that the polymer atoms in $\xi_{t}$ survive for all time, which implies Theorem 1.3.

As remarked in the Introduction after Theorem 1.4, the concentration of monomers will, in fact, be very low when $m$ and $\lambda$ are large. We outline the argument, which is a minor adaptation of the one just given for Theorem 1.3. Specifically, one needs to replace (4.5) and (4.6) with analogous statements, but where $L^{\prime}=1$ and $\varepsilon \rightarrow 0$ as $\delta \rightarrow 0$. (The reasoning for these variants is the same as that for the original versions.) On the other hand, the bound in (5.2) holds for any given $\delta>0$, if $m$ is chosen large enough. So, use of the same reasoning as in the above paragraphs shows that (5.4), in fact, holds with $L^{\prime}=1$. Consequently, there are, with overwhelming probability, polymer atoms in the corresponding $M \times M$ square for a given $B_{1}^{z}$ in the above cone, for large $k$. Since there can be no monomers in such a square, this shows that the density of monomers goes to 0 as $m \rightarrow \infty$, as desired. (Not surprisingly, the actual rate at which this convergence occurs will be much faster than the bounds employed in the above reasoning.)

The basic idea behind the proof of Proposition 5.1 goes as follows. Except on a set of small probability, "not very many" monomers have time to bond to $\hat{B}=\hat{B}(0)$ over $(0,2 T]$. Also, if $B(z),\|z\|_{1}=1$, is initially occupied, there can be no monomers in $B$ then, since all such sites are within range $R$ of one another. Because $\lambda$ is large, there is thus plenty of time and space for sufficiently many polymers to bond to $B$ over $(0, T]$ for $B$ to remain occupied over $[T, 2 T]$.

To show that this scenario in fact holds, we employ the following events:

$$
\begin{aligned}
F & =\left\{\text { at most } \frac{1}{70} m^{2} \text { monomers attempt to bond to } \hat{B} \text { over }(0,2 T]\right\}, \\
G_{1} & =\left\{\text { there exist at least } \frac{1}{15} m^{2} \text { polymer atoms in } B \text { at some } t \in[0, T]\right\}, \\
G_{2} & =\left\{\text { at least } \frac{\lambda M^{2}}{12,000} \text { polymer atoms bond to } B \text { over }(0, T]\right\} .
\end{aligned}
$$

Let $\tau$ denote the time at which the event $G_{1}$ first occurs, and set $G=G_{1} \cup G_{2}$. Also, let $H_{1,2}(0)$ denote the event that $B(0)$ is occupied over all of $[T, 2 T]$.

To demonstrate Proposition 5.1, we employ the following lemmas.

LEMMA 5.5. For appropriate $c_{1}>0$,

$$
P\left(F^{c}\right) \leq \exp \left\{-c_{1} m^{2}\right\}
$$


LEMMA 5.6. For appropriate $c_{2}>0$ and large $m$,

$$
P\left(F \cap G^{c}\right) \leq \exp \left\{-c_{2} \lambda m^{2}\right\}
$$

under $H_{0}(z)$ for some $z$ with $\|z\|_{1}=1$.

LEMMA 5.7. For large $\lambda$,

$$
H_{1,2}^{c}(0) \subset F^{c} \cup G^{c} .
$$

Proposition 5.1 follows quickly from Lemmas 5.5-5.7. By Lemma 5.7,

$$
P\left(H_{1,2}^{c}(0)\right) \leq P\left(F^{c}\right)+P\left(F \cap G^{c}\right) .
$$

Plugging Lemmas 5.5 and 5.6 into the right side of (5.8) gives

$$
P\left(H_{1,2}^{c}(0)\right) \leq \exp \left\{-c_{3} m^{2}\right\},
$$

under $H_{0}(z),\|z\|_{1}=1$, large $m$ and $\lambda$, and appropriate $c_{3}>0$. This clearly implies (5.2). So, to prove Proposition 5.1, it suffices to show Lemmas 5.5-5.7.

We first recall the following elementary large deviations estimate, which will also be used in later sections. If $X$ is a Poisson random variable with mean $\Lambda$, then it has moment generating function $\exp \{\Lambda(\exp (t)-1)\}$. Employment of Chebyshev's inequality, for small enough $t$, shows that for $\eta \geq 2 / 5$ (any positive number will do) and appropriate $c_{4}>0$,

$$
\begin{aligned}
& P(X \geq(1+\eta) \Lambda) \leq \exp \left\{-c_{4} \Lambda\right\}, \\
& P(X \leq(1-\eta) \Lambda) \leq \exp \left\{-c_{4} \Lambda\right\} .
\end{aligned}
$$

ProOF OF LeMma 5.5. The result follows almost immediately from the first half of (5.10) with $\eta=1$ and $\Lambda=m^{2} / 40$. Since $|\hat{B}| \leq 7 R^{2}$, for large $R$, and $|(0,2 T]|=1 / 1,000 N^{2}$, the number of monomers which attempt to bond to $\hat{B}$ over $(0,2 T]$ is Poisson with mean at most $7 R^{2} / 1,000 N^{2} \leq m^{2} / 140$.

Proof of LeMma 5.6. For $\|z\|_{1}=1$, every site in $B(z)$ is within distance $2 M \leq R$ of every site in $B$. Since under $H_{0}(z), B(z)$ contains polymer atoms at time $0, B$ has no monomers then. Because $m^{2} / 70 \leq M^{2} / 3 N^{2}$ for large $m$, it follows that under $F \cap H_{0}(z)$, there are, over [0,T], never more than $M^{2} / 3 N^{2}$ monomers in $B$. Likewise, under $G_{1}^{c}$, there are never more than $m^{2} / 15 \leq M^{2} / 3 N^{2}$ polymer atoms in $B$. Since monomers and polymers cannot coexist in $B$, it thus follows that under $F \cap G_{1}^{c} \cap H_{0}(z)$, there are, over $[0, T]$, never more than $M^{2} / 3 N^{2}$ occupied sites in $B$. Denoting this last event by $K$, one has

$$
F \cap G_{1}^{c} \cap H_{0}(z) \subset K .
$$

An occupied site $y$ can obstruct the bonding of a polymer at $x+[0, N)^{2}$ only if $y \in x+[0, N)^{2}$. When there are at most $M^{2} / 3 N^{2}$ occupied sites in $B$, polymers at at most $M^{2} / 3$ positions are obstructed by them. Since $M$ is assumed to be large compared with $N$, there remain at least $M^{2} / 3$ positions in which polymers can bond so as to be completely in $B$. Over these times, 
polymers therefore bond inside $B$ at rate at least $\lambda M^{2} / 3$. Now, assume any initial configuration. Employing the second half of (5.10) with $\eta=1 / 2$, it follows that the probability that there are, over $[0, T]$, never more than $M^{2} / 3 N^{2}$ occupied sites, and that fewer than $\lambda M^{2} / 12,000 N^{2}$ polymers (and hence $\lambda M^{2} / 12,000$ polymer atoms) bond in $B$ over $(0, T]$ is at most

$$
\exp \left\{-\frac{c_{4} \lambda M^{2}}{6,000 N^{2}}\right\} \leq \exp \left\{-c_{2} \lambda m^{2}\right\}
$$

for appropriate $c_{2}>0$. That is,

$$
P\left(G_{2}^{c} \cap K\right) \leq \exp \left\{-c_{2} \lambda m^{2}\right\} .
$$

Lemma 5.6 follows from (5.11) and (5.12).

ProOF OF LemMa 5.7. To demonstrate the lemma, it suffices to show that

$$
F \cap G_{1} \subset H_{1,2}(0)
$$

and

$$
F \cap G_{2} \subset H_{1,2}(0) .
$$

Under $G_{1}$, there are least $m^{2} / 15$ polymer atoms in $B$ at the time $\tau \in[0, T]$. Only those monomers bonding in $\hat{B}$ over $(0,2 T]$ can interact with these polymer atoms; under $F$, there are at most $\mathrm{m}^{2} / 70$ such monomers. It follows that, under $F \cap G_{1}$, there are at least $m^{2} / 20$ polymer atoms in $B$ at all times in $[T, 2 T]$. So, (5.13) holds.

Under $G_{2}$, at least $\lambda M^{2} / 12,000$ polymer atoms bond to $B$ over $(0, T]$. Only those monomers in $\hat{B}$ at time 0 or bonding to $\hat{B}$ over $(0,2 T]$ can interact with these polymer atoms. Under $F$, there are at most

$$
7 R^{2}+\frac{1}{70} m^{2} \leq 8 R^{2}
$$

such monomers. For large $\lambda$,

$$
\frac{\lambda M^{2}}{12,000}-8 R^{2} \geq \frac{m^{2}}{20}
$$

Thus, under $F \cap G_{2}$, there are at least $m^{2} / 20$ polymer atoms in $B$ at all times in $[T, 2 T]$. So, (5.14) also holds.

We note briefly that (5.3) can be extended to (4.8) without too much difficulty by reasoning along the lines of the end of Section 4 . The bound for monomer bonding in $\hat{B}$ in the proof of Lemma 5.5 holds, irrespective of the data on the boundary of $\hat{B}$. One can also proceed in the proof of Lemma 5.6 by observing that, under $K$, there are always at least $M^{2} / 3$ positions in which polymers can bond. The polymer bonding times $\nu_{n}^{x}$ for the "first" $M^{2} / 3$ of these positions can therefore be coupled together so that systems corresponding to different boundary data share the same exceptional event in (5.12). The bounds corresponding to (5.13) and (5.14) continue to hold in the multidimensional setting as well. 
6. Monomer survival for large $m$ and $N$. Basic setup. It was shown in Sections 2 and 3 that either a short range of interaction or low bonding rate for polymers ensures poisoning by monomers, and in Section 5 that polymer atoms survive for a long range of interaction and high bonding rate. In Sections 6-8, we demonstrate Theorem 1.4, which states that for a long range of interaction and large polymer size, monomers survive. The assumption on the range is presumably not necessary, especially in view of Theorem 1.1. Some assumption on the polymer size is needed, though, on account of possible polymer poisoning as in the ZGB model. There are no restrictions on the polymer bonding rate, although, as we shall see, the argument becomes more complicated when the rate is large.

The basic reason for the survival of monomers under these conditions is the inability of polymers to completely "seal off" large regions where monomer bonding would be prevented. In regions where monomers are able to bond (either in relatively large open regions or in "cracks" between polymers), they will interact with nearby polymer atoms. Suppose, for instance, that the interaction is with a polymer atom which is surrounded within distance $N$ by other polymer atoms. The vacant site which is created in this case cannot be occupied by a polymer atom until on the order of $N^{2}$ nearby polymer atoms are removed, so that a polymer is capable of bonding. Before all of these polymer atoms are removed, there will be substantial opportunity for more monomers to bond nearby. This can create vacant sites in additional pure polymer regions which cannot be filled immediately by polymers, and so on. Under this scenario, polymer atoms should not be able to poison $\mathbf{Z}^{2}$. Rather, there should be enough open space for bonding monomers to "strike back," although these monomers may only on occasion stick to $\mathbf{Z}^{2}$. It is also, of course, possible for monomers, if $\lambda$ is small enough, to eventually poison $\mathbf{Z}^{2}$, as in Theorem 1.2.

It is unfortunately quite cumbersome to make the above scenario mathematically precise. One needs to be able to characterize "good" configurations that are resistant to local polymer poisoning, and to specify a mechanism under which the evolution of the system can be monitored. (A set will be referred to here as poisoned if it is completely occupied by monomer or by polymer atoms.) Since the geometry of allowable configurations is fairly complicated, and the process $\xi_{t}$ is nonattractive, considerable care must be exercised. For our argument, we need to distinguish two cases, where $\lambda \leq \delta m^{2}$ and where $\lambda>\delta \mathrm{m}^{2}$, for appropriately small $\delta>0$. Loosely speaking, a good configuration on an $M \times M$ square will be one in which either (i) the number of polymer atoms which need to be removed from the square before it is possible to poison it with polymer atoms is large ( $\geq \varepsilon_{1}\left(m^{2} \wedge N^{2}\right)$, appropriate $\varepsilon_{1}>0$ ); or (ii) there are enough vacant sites to make it impossible for polymer atoms to poison the square over a moderate period of time or, in the case $\lambda \leq \delta m^{2}$, (iii) there is at least one monomer in the square.

As in Section 5, we set $M=2 \mid R / 4](\approx R / 2)$, where $R$ is the range of $\xi_{t}$. Using $M \times M$ squares $B(z)$ as specified in (4.7), we can partition $\mathbf{Z}^{2}$ as 
before. The square $B(z)$ will be said to be occupied when either (i), (ii) or (iii) above is satisfied for $\lambda \leq \delta m^{2}$, and when (i) or (ii) is satisfied for $\lambda>\delta m^{2}$. We will show that if $B(z)$ is occupied, then, after elapsed time $T=1 / \delta$, its immediate neighbors are occupied with probability close to 1 . This will allow us to employ the rescaling algorithm from Section 4 to conclude that monomers survive for all time. We divide the work that is required into three parts. Proposition 6.3 is one of the main tools; since the proof requires substantial preparation, we postpone it to Section 7. Using Proposition 6.3, we will demonstrate monomer survival for $\lambda \leq \delta m^{2}$ in this section. In Section 8 , we cover the case where $\lambda>\delta m^{2}$. There, the argument becomes more technical, but can be built on the work in this section.

We begin by introducing notation needed to formulate the condition (i) above and Proposition 6.3. Because of the translation invariance of the evolution of $\xi_{t}$, it is enough to focus on the set $B=B(0)=B_{M}^{0}$. By $B^{I_{j}}$, we will mean those $x \in B$ for which $d\left(B^{c},\{x\}\right) \geq j N$, where the distance function $d(\cdot, \cdot)$ is in terms of the $L^{\infty}$ norm. Note that for large $m$ and fixed $j$, $\left|B^{I_{j}}\right| \approx|B|$. Consider now a configuration $\xi$ with $\xi(x)=0$ for a given $x \in \mathbf{Z}^{2}$, and $\xi(y) \neq 1$ for all $y \in B_{2 N}^{x}$. Let $\Gamma_{x}(\xi)$ denote the minimal number of polymer atoms one needs to remove from $\xi$ so that a polymer occupying $x$ can bond. This procedure entails creating a vacant $N \times N$ square containing $x$; therefore, any choice of the corresponding $\Gamma_{x}(\xi)$ sites will be contained within $B_{2 N}^{x}$. Also, set

$$
\hat{\Gamma}_{x}(\xi)=\Gamma_{x}(\xi) \wedge\left\lfloor\frac{N^{2}}{2}\right\rfloor .
$$

For any configuration $\xi$, let $\mathscr{A}(\xi)$ denote the collection of sets $A=$ $\left\{x_{1}, \ldots, x_{n}\right\}$ with $x_{i} \in B^{I_{1}},\left\|x_{i_{1}}-x_{i_{2}}\right\|_{\infty} \geq 2 N$, and $\Gamma_{x_{i}}(\xi)>0$. We define the "counter" for $\xi$,

$$
K(\xi)=\max _{A \in \mathscr{A}(\xi)} \sum_{x_{i} \in A} \hat{\Gamma}_{x_{i}}(\xi)
$$

The purpose of $K(\xi)$ is to provide a manipulable lower bound on the number of polymer atoms which need to be removed before $B$ can be completely filled by polymer atoms. (It follows easily from the definition of $\mathscr{A}(\xi)$ that $K(\xi)$ is a lower bound.) The condition that $K(\xi) \geq \varepsilon_{1}\left(m^{2} \wedge N^{2}\right), \varepsilon_{1}>0$, is the condition referred to in (i) earlier. The reason for the specific choice of $K(\xi)$ using $\hat{\Gamma}_{x_{i}}(\xi)$ comes from Lemma 7.14 in the proof of Proposition 6.3.

We need to specify what is meant for a site to be surrounded by polymer atoms. Denote by $D(\xi)$ the set of points $x \in B^{I_{1}}$ for which $\xi(y)=2$ for all $y \in B_{2 N / 3}^{x}$. In general, we shall refer to the subset $B_{N / 3}^{x}$ as the core of $B_{N}^{x}$. Note that when a polymer $P$ bonds at time $t$ and none of its atoms interacts immediately with a monomer, then those sites in the core of $P$ are in $D\left(\xi_{t}\right)$, and so

$$
\left|P \cap D\left(\xi_{t}\right)\right| \geq\left(\left\lfloor\frac{N}{3}\right\rfloor\right)^{2}
$$


Having defined $K$ and $D$, we can now state Proposition 6.3. As mentioned earlier, the proof will be given in Section 7. Here and later on, a parameter $\beta>0$ will be said to be large relative to another parameter $\alpha>0$ when $\beta / \alpha$ is bounded away from 0 .

Proposition 6.3. Assume that $\left|D\left(\xi_{0}\right)\right| \geq \varepsilon R^{2}$, where $\varepsilon>0$, and that $m$ and $N$ are large relative to $1 / \varepsilon$. Assume further that $K\left(\xi_{0}\right) \geq a$ for some $a \leq \varepsilon\left(m^{2} \wedge N^{2}\right) / 128$. Then, for all $\lambda$ and appropriate $C_{1}$ and $C_{2}>0$,

$$
P\left(K\left(\xi_{t}\right) \leq \frac{a}{2} \text { at some } t \leq \frac{\varepsilon}{10^{5}}\right) \leq C_{1} \exp \left\{-C_{2} a\right\}
$$

and

$$
P\left(K\left(\xi_{\varepsilon / 10^{5}}\right) \leq 2 a\right) \leq C_{1} \exp \left\{-C_{2} a\right\} .
$$

Any multiple of $a$ can be chosen in (6.5). We require just the bound $a$ in this section; the bound $2 a$ will be used in Section 8 .

We next introduce the notation we need to formulate condition (ii) given earlier, in the case where $\lambda \leq \delta m^{2}$. Let $\xi$ be a configuration with no monomers in $B$, and let $L(\xi)$ denote the maximal number of vacant $N \times N$ squares $B_{N}^{y_{1}}$ with $\left\|y_{l_{1}}-y_{l_{2}}\right\|_{\infty} \geq 7 N$ and $B_{N}^{y_{1}} \subset B^{I_{3}}$. Also, let $\mathscr{P}(\xi)=$ $\left\{P_{1}(\xi), \ldots, P_{L(\xi)}(\xi)\right\}$ be a choice of such squares. We choose the random collection $\mathscr{P}\left(\xi_{t}\right)$ to remain constant except when $\xi_{t}$ changes in $B$. For $\xi \equiv 0$ and $m$ large, it is not hard to see that $L(\xi) \geq m^{2} / 225$. The presence of a large collection $\mathscr{P}(\xi)$ will allow monomers, as we will later show, enough time to bond and interact with polymer atoms in $D(\xi)$ before $B$ can completely fill up with polymers. [In Section 8 , when handling the case $\lambda>\delta \mathrm{m}^{2}$, we will need to decompose $\mathscr{P}(\xi)$ into "quick" and "slow" subsets, $\mathscr{Q}(\xi)$ and $\mathscr{S}(\xi)$.]

Consider now the process $\xi_{t}$, with $\lambda \leq \delta m^{2}$, where $\delta>0$ will be specified later. We will say that $B$ is occupied for the configuration $\xi$ if either (1) $K(\xi) \geq \varepsilon_{1}\left(m^{2} \wedge N^{2}\right)$, where $\varepsilon_{1}=1 / 8,000$, (2) $L(\xi) \geq \varepsilon_{1} m^{2}$ or $(3) \xi(x)=$ 1 for some $x \in B$. (Any smaller choice of $\varepsilon_{1}$ also works.) We will find it convenient later on to say that $B$ is well occupied if one of the same three conditions holds, but with $\varepsilon_{1}$ in the lower bounds in (1) and (2) being replaced by $2 \varepsilon_{1}$. We set $\bar{\varepsilon}_{1}=\varepsilon_{1}$ or $\bar{\varepsilon}_{1}=2 \varepsilon_{1}$, depending on the context. The set $B(z)$ will be occupied if one of the corresponding three conditions for the translates of $K(\xi), L(\xi)$ and $\xi$ holds; let $H_{k}(z)$ denote this event for $\xi_{k T}$. The main result in this section is the following analog of Proposition 5.1

Proposition 6.6. For small enough $\delta>0, \lambda \leq \delta m^{2}, T=1 / \delta$ and $m^{2} \wedge$ $N^{2}$ large relative to $1 / \delta$,

$$
P(B(0) \text { is not occupied at some } t \in[T, 2 T]) \leq \delta
$$

if $B(z)$ is occupied at time 0 for some $z$ with $\|z\|_{1}=1$. 
To obtain Theorem 1.4 for $\lambda \leq \delta m^{2}$, we employ the rescaling algorithm provided in Section 4 and already used in Section 5 . We observe that by Proposition 6.6,

$$
P\left(H_{k+1}^{c}(z) \mid \mathscr{F}_{k T}\right) \leq \delta
$$

under $H_{k}\left(z^{\prime}\right)$ for any $z^{\prime}$ with $\left\|z-z^{\prime}\right\|_{1}=1$. As mentioned at the end of Section 4, we, in fact, require the multidimensional version given in (4.8) to show survival of monomer atoms. For this, we set

$$
\hat{B}(z)=B_{3 M+2 R}^{z}
$$

here, which is the set of all points within distance $M+R$ of $B(z)$. In order to derive (6.8), and, more generally (4.8), we will need to analyze the behavior of $\xi_{t}$ in $B(0)$ and $B(z),\|z\|_{1}=1$, and to employ an upper bound on the number of monomers that bond at sites within distance $R$ of these sets. [These sites are all in $\hat{B}(0)$.] Including a boundary region of width $R$ around $\hat{B}(0)$, to separate it from other squares $\hat{B}(z)$, gives a square of length $3 M+4 R \leq 12 M$ for large $R$. So, we stipulate that $\left\|z_{a_{1}}-z_{a_{2}}\right\| \geq 12$ in (4.8). Reasoning as at the end of Section 4 gives the independence of the different squares needed for (4.8) to hold. As in Section 5, given (4.8), one can then compare the process $V_{k}$ in (4.9) with the corresponding oriented percolation $W_{k}$ by (4.10). This enables us to employ the bound (4.6). The assumption that $\xi_{0} \in \Xi_{1}$ in Theorem 1.4 implies that at any $t_{1}>0$, the number of occupied squares $B(z)$ is infinite with probability 1 . Restarting the process at time $t_{1}$ and using the same comparisons as in Section 5, the analog of (5.4) then follows, as does the extension of the survival of monomers to nonlattice times. This implies Theorem 1.4 for $\lambda \leq \delta m^{2}$ and appropriate $\delta>0$.

Demonstration of Proposition 6.6. The idea behind the proof of the proposition is as follows. We will first show that for any configuration $\xi$ without monomers in $B$, either $K(\xi)$ or $L(\xi)$ is large [in which case $B$ is occupied (well occupied)], or $|D(\xi)|$ is large (Lemma 6.9). If $B$ is occupied (or well occupied) at time 0 , we can therefore stop the process $\xi_{t}$ at a random time $S$ while $\left|D\left(\xi_{t}\right)\right|$ is large, but just before both $K\left(\xi_{t}\right)<\left[\bar{\varepsilon}_{1}\left(m^{2} \wedge N^{2}\right)\right]$ and $L\left(\xi_{t}\right)$ $<\left\lfloor\bar{\varepsilon}_{1} m^{2}\right\rfloor$. In the case where $L\left(\xi_{S}\right)=\left\lfloor\bar{\varepsilon}_{1} m^{2}\right\rfloor$, one can show that at time $S^{\prime}=S+\varepsilon_{2}, \varepsilon_{2}=\left(16 \delta R^{2}\right)^{-1}$, with probability close to $1,\left|D\left(\xi_{S^{\prime}}\right)\right|$ is large and $K\left(\xi_{S^{\prime}}\right) \geq \bar{\varepsilon}_{1}\left(m^{2} \wedge N^{2}\right)$ (Proposition 6.16). In the other case, where $K\left(\xi_{S}\right)=$ $\left\{\bar{\varepsilon}_{1}\left(m^{2} \wedge N^{2}\right)\right]$, these bounds already hold at time $S$. [These events are given in (6.24)]. Set $S^{\prime \prime}=S^{\prime}$ or $S^{\prime \prime}=S$, depending on the case. At time $\sigma=S^{\prime \prime}+\varepsilon_{3}$, with $\varepsilon_{3}=1 /\left(3 \cdot 10^{6}\right)$, one obtains that, with probability close to $1, K\left(\xi_{\sigma}\right)>$ $\bar{\varepsilon}_{1}\left(m^{2} \wedge N^{2}\right)$ [(6.5) of Proposition 6.3], in which case $\xi_{\sigma}$ is occupied (or well occupied). Also, with probability close to $1, K\left(\xi_{t}\right)>\bar{\varepsilon}_{1}\left(m^{2} \wedge N^{2}\right) / 2$ over $t \in\left[S^{\prime \prime}, \sigma\right][(6.4)$ of Proposition 6.3]. Together, these observations imply that, with probability close to $1, B$ is not poisoned over $[0, \sigma]$ and is occupied at time $\sigma$ if $B$ is initially occupied (Proposition 6.31). Similarly, $B$ is occupied over $[0, \sigma]$ and is well occupied at time $\sigma$ if $B$ is initially well occupied. 
Iterating the above procedure on an occupied square $B(z),\|z\|=1$, at most $2 T / \varepsilon_{3}$ times, it follows that, over [0,2T], $B(z)$ is, with probability close to 1 , not poisoned by polymer atoms [Proposition (6.36)]. This allows monomers to continually bond in $B(z)$. It is not difficult to check that, under this assumption on $B(z)$, the neighboring square $B=B(0)$ will be well occupied at some $\tau \leq T$ (proof of Proposition 6.6). One can therefore also apply the above iterative procedure to the well-occupied square $B$. One concludes that with probability close to $1, B$ is always occupied over $[\tau, \tau+2 T] \supset[T, 2 T]$. This demonstrates the claim in Proposition 6.6.

Most of the technical work in deriving Proposition 6.6 is done in Proposition 6.3, Lemma 6.9 and Proposition 6.16. As previously mentioned, the proof of Proposition 6.3 is deferred to Section 7. Lemma 6.9 and its proof are given below. The following two lemmas, Lemmas 6.13 and 6.14, are used for Proposition 6.16.

In Lemma 6.9, we show that if there are no monomers present in $B$, and if both $K(\xi)$ and $L(\xi)$ are not large, then $|D(\xi)|$ must be large. Here, $P_{l}^{j}$ will stand for the $j N \times j N$ square centered around the $N \times N$ square $P_{l}$, and $\mathscr{P}^{j}(\xi)=\left\{P_{1}^{j}(\xi), \ldots, P_{L(\xi)}^{j}(\xi)\right\}$ the corresponding collection, where $P_{1}(\xi), \ldots, P_{L(\xi)}(\xi)$ and $\mathscr{P}(\xi)$ are as defined earlier in the section. Here and later on in the paper, we implicitly assume that $m$ and $N$ are chosen large enough so that certain nuisance constants can be absorbed into bounds by an appropriate change in the corresponding factor (for instance, so that the fractional part of $R / 4$ in the definition of $M$ can be neglected, and also, so that $\left|B^{I_{5}}\right| \approx|B|$ can be employed).

LEMMA 6.9. Assume that $B$ contains no monomers for a given configuration $\xi$, and that $K(\xi) \leq m^{2} / 400$ and $L(\xi) \leq m^{2} / 4,000$. Then,

$$
|D(\xi)| \geq \frac{R^{2}}{15} .
$$

Proof. Let $A=\left\{x_{1}, \ldots, x_{n}\right\} \in \mathscr{A}(\xi)$ be a set as in (6.2), on which $K(\xi)$ is achieved. We claim that if there are no monomers in $B$, then

$$
B^{I_{5}} \subset \bigcup_{i=1}^{n} B_{5 N}^{x_{i}} \cup \bigcup_{l=1}^{L(\xi)} P_{l}^{15}(\xi) \cup D(\xi) .
$$

Assuming (6.11), it is not difficult to show (6.10). Note that

$$
\left|\bigcup_{i=1}^{n} B_{5 N}^{x_{i}}\right| \leq 25 n N^{2} \text { and }\left|\bigcup_{l=1}^{L(\xi)} P_{l}^{15}(\xi)\right| \leq 225 L(\xi) N^{2} .
$$

Also, $\left|B^{I_{5}}\right| \geq R^{2} / 5$ for large $m$. So, by (6.11),

$$
\frac{R^{2}}{5} \leq 25 n N^{2}+225 L(\xi) N^{2}+|D(\xi)| .
$$

Since $n \leq K(\xi) \leq m^{2} / 400, L(\xi) \leq m^{2} / 4,000$ and $R=m N$, (6.10) follows from (6.12). 
To show (6.11), suppose that $x \in B^{I_{5}}$ but $x \notin \cup_{i=1}^{n} B_{5 N}^{x_{i}} \cup D(\xi)$. Since $x \notin D(\xi)$, there is a $y \in B^{I_{4}}$ with $\xi(y)=0$ and $\|y-x\|_{\infty} \leq N / 3$. Since $x \notin B_{5}^{x} \dot{N}$ implies that $\left\|x-x_{i}\right\|_{\infty} \geq \frac{5}{2} N$, it follows that for all $i$,

$$
\left\|y-x_{i}\right\|_{\infty} \geq\left\|x-x_{i}\right\|_{\infty}-\|y-x\|_{\infty} \geq 2 N .
$$

If $\Gamma_{y}(\xi) \neq 0$ were true, then $\tilde{A}=A \cup\{y\} \in \mathscr{A}(\xi)$ would give a greater value of $K(\xi)$ in (6.2), which would be a contradiction. So, $\Gamma_{y}(\xi)=0$. We claim this implies that $x \in \cup_{l=1}^{L(\xi)} P_{l}^{15}(\xi)$. To see this, assume the contrary. Letting $y_{1}, \ldots, y_{L(\xi)}$ denote the centers of $P_{1}(\xi), \ldots, P_{L(\xi)}$, one has $\left\|x-y_{l}\right\|_{\infty} \geq$ $15 N / 2$, and so

$$
\left\|y-y_{l}\right\|_{\infty} \geq 7 N \text { for all } l \text {. }
$$

Since $B_{N}^{y} \subset B^{I_{3}}$, this implies that $\mathscr{P}(\xi)$ is not maximal. So, in fact, $x \in$ $\cup_{l=1}^{L(\xi)} P_{l}^{15}(\xi)$, which implies (6.11).

Suppose that the polymer atom at $y \in D\left(\xi_{0}\right)$ desorbs over $(0, t]$. Since $y$ was originally surrounded by polymer atoms, $\hat{\Gamma}_{y}\left(\xi_{t}\right)$, and hence $K\left(\xi_{t}\right)$, must remain large until a substantial number of other polymer atoms desorb from $B$. This is the content of Lemma 6.13. Here and later on, we set $\bar{B}=B_{M+2 R}^{0}$, the set of all sites within distance $R$ of $B$; clearly, only monomers in $\bar{B}$ can interact with polymer atoms in $B$. One can check that $|\bar{B}| \leq 7 R^{2}$. Also, let $Z_{1}(t)$ denote the number of monomers that have bonded in $\bar{B}$ by time $t$.

LEMMA 6.13. Suppose that $y \in D\left(\xi_{0}\right)$ and $\xi_{s}(y)=0$ for some $s \in(0, t]$. For $Z_{1}(t)<N^{2} / 20$, one has $\hat{\Gamma}_{y}\left(\xi_{t}\right)>N^{2} / 20$ and hence $K\left(\xi_{t}\right)>N^{2} / 20$.

Proof. Any $N \times N$ square containing $y$, when intersected with $B_{2}^{y}{ }_{N / 3}$, contains a square $B_{N / 3}^{z}$. Since for $y \in D\left(\xi_{0}\right), B_{2 N / 3}^{y}$ is initially completely occupied by polymer atoms, $B_{N / 3}^{z}$ has more than $N^{2} / 20$ such atoms when $Z_{1}(t)<N^{2} / 20$. (Choose $N$ large enough so that the number of sites lost through noninteger $N / 6$ is negligible). So, $\hat{\Gamma}_{y}\left(\xi_{t}\right)>N^{2} / 20$ under $Z_{1}(t)<$ $N^{2} / 20$ and $\xi_{s}(y)=0$. Since $K\left(\xi_{t}\right) \geq \hat{\Gamma}_{y}\left(\xi_{t}\right)$ for $y \in B^{I_{1}}, K\left(\xi_{t}\right)>N^{2} / 20$ also follows.

A bonding polymer can intersect an $N \times N$ square in $4 N^{2}$ different positions. Under the assumption $\lambda \leq \delta m^{2}$, this implies that the rate at which a vacant $N \times N$ square is intersected by a polymer is at most $4 \delta R^{2}$. Consider an initial configuration $\xi$ for which $L(\xi) \geq L_{0}$. Let $Z_{2}(t)$ denote the number of the squares $P_{1}\left(\xi_{0}\right), \ldots, P_{L_{0}}\left(\xi_{0}\right)$ which remain free of polymer atoms over $[0, t]$. Using the above upper bound on $\lambda$, we obtain the following lower bound on $Z_{2}(t)$. [Recall that $\varepsilon_{2}=\left(16 \delta R^{2}\right)^{-1}$.]

Lemma 6.14. Assume that $L(\xi) \geq L_{0}$ for a given $L_{0}$, and that $t \leq \varepsilon_{2}$ for $\lambda \leq \delta m^{2}$. Then,

$$
P\left(Z_{2}(t) \leq \frac{L_{0}}{2}\right) \leq \exp \left\{-\frac{c_{4} L_{0}}{4}\right\},
$$

where $c_{4}>0$ is chosen as in (5.10). 
Proof. The rate at which polymers intersect $\bigcup_{l=1}^{L_{0}} P_{l}\left(\xi_{0}\right)$ is at most $4 \delta L_{0} R^{2}$. So, the number of squares $P_{l}\left(\xi_{0}\right)$ which contain polymer atoms at some time in $[0, t]$ is dominated by a Poisson random variable with mean $4 \delta L_{0} R^{2} t$. For $t \leq 1 / 16 \delta R^{2}$, this is at most $L_{0} / 4$. So, (6.15) follows from (5.10) with $\eta=1$. [Note that, along the lines of the discussion at the end of Section 4 on the coupling of different systems, the derivation of the bound in (6.15) depends on $L_{0}$ rather than on the precise configuration of $\xi_{t}$ in $B$. This allows one to couple the corresponding exceptional sets in Proposition (6.16).]

Proposition 6.16 enables us to control the behavior of $\xi_{t}$ over a short interval of time when $\left|D\left(\xi_{0}\right)\right|$ and $L\left(\xi_{0}\right)$ are large. It says that over this interval, $L\left(\xi_{t}\right)$ typically remains large, whereas at the end, $K\left(\xi_{t}\right)$ and $\left|D\left(\xi_{t}\right)\right|$ are large. Here, $V_{A}(\xi)$ denotes the number of vacant sites in the set $A$.

Proposition 6.16. Assume that $L\left(\xi_{0}\right) \geq \varepsilon m^{2}$ for some $\varepsilon>0$ and that $\lambda \leq \delta m^{2}$. Then, for appropriate $C_{3}>0$,

$$
P\left(L\left(\xi_{t}\right) \leq \frac{\varepsilon m^{2}}{2} \text { at some } t \leq \varepsilon_{2}\right) \leq \exp \left\{-C_{3} \varepsilon m^{2}\right\},
$$

where $\varepsilon_{2}=\left(16 \delta R^{2}\right)^{-1}$. Assume further that $\left|D\left(\xi_{0}\right)\right| \geq \varepsilon^{\prime} R^{2}$ for some $0<$ $\varepsilon^{\prime}<1 / 10$, and that $\varepsilon^{\prime}\left(m^{2} \wedge N^{2}\right)$ is large relative to $1 / \delta$. Then,

$$
P\left(K\left(\xi_{\varepsilon_{2}}\right) \leq \frac{N^{2}}{20} \text { or }\left|D\left(\xi_{\varepsilon_{2}}\right)\right| \leq \frac{\varepsilon^{\prime} R^{2}}{2}\right) \leq 3 \exp \left\{-\frac{C_{3} \varepsilon \varepsilon^{\prime}}{\delta}\right\}
$$

Proof. We set $L_{0}=\varepsilon m^{2}$ and define $Z_{2}(t)$ as in Lemma 6.14. It is immediate that $Z_{2}(t) \leq L\left(\xi_{t}\right)$ for all $t$; the inequality (6.17) therefore follows from (6.15). Denoting the complement of the exceptional set in (6.17) by $G_{1}$, it follows that on $G_{1}$,

$$
V_{B}\left(\xi_{t}\right) \geq \frac{\varepsilon R^{2}}{2} \text { for } t \leq \varepsilon_{2}
$$

There are fewer than $5 R^{2}$ sites within range $R$ of a given site. On account of (6.19) and $\left|D\left(\xi_{0}\right)\right| \geq \varepsilon^{\prime} R^{2}$, the rate of desorption from $D\left(\xi_{0}\right)$ until the first such desorption occurs is therefore at least

$$
\frac{\varepsilon R^{2}}{2} \varepsilon^{\prime} R^{2} \frac{1}{5 R^{2}}=\frac{\varepsilon \varepsilon^{\prime} R^{2}}{10}
$$

on $G_{1}$. Let $G_{2}$ be the event some polymer atom desorbs from $D\left(\xi_{0}\right)$ by time $\varepsilon_{2}$. Under $G_{1}$, the probability of $G_{2}^{c}$ occurring is thus at most exponential with parameter

$$
\frac{\varepsilon \varepsilon^{\prime} R^{2}}{10} \frac{1}{16 \delta R^{2}}=\frac{\varepsilon \varepsilon^{\prime}}{160 \delta}
$$


Together with (6.17), this shows that

$$
P\left(G_{2}^{c}\right) \leq \exp \left\{-C_{3} \varepsilon m^{2}\right\}+\exp \left\{-\frac{\varepsilon \varepsilon^{\prime}}{160 \delta}\right\} .
$$

(Note that, along the lines of the discussion at the end of Section 4 , the above bounds do not depend on the precise configuration of $\xi_{t}$ in $B$, but only on the above rates. So, the corresponding exceptional sets may be coupled.)

By Lemma $6.13, K\left(\xi_{\varepsilon_{2}}\right)>N^{2} / 20$ on $G_{2}$, unless $N^{2} / 20$ monomers have bonded in $\bar{B}$ by then. Since the rate of bonding of monomers in $\bar{B}$ is trivially at most $7 R^{2}, Z_{1}\left(\varepsilon_{2}\right)$ is bounded above by a Poisson random variable with mean $7 / 16 \delta$. It follows from (5.10), with $\eta=1$, that for fixed $\delta$, and for $\varepsilon^{\prime}\left(m^{2} \wedge N^{2}\right)$ large relative to $1 / \delta$,

$$
P\left(Z_{1}\left(\varepsilon_{2}\right) \geq \frac{\varepsilon^{\prime}}{2}\left(m^{2} \wedge N^{2}\right)\right) \leq \exp \left\{-\frac{\varepsilon^{\prime} c_{4}}{4}\left(m^{2} \wedge N^{2}\right)\right\} .
$$

Since $N^{2} / 20>\varepsilon^{\prime}\left(m^{2} \wedge N^{2}\right) / 2$, it follows from (6.20) and (6.21), that

$$
P\left(K\left(\xi_{\varepsilon_{2}}\right) \leq \frac{N^{2}}{20}\right) \leq 3 \exp \left\{-\frac{C_{3} \varepsilon \varepsilon^{\prime}}{\delta}\right\},
$$

where we rechoose $C_{3}$ if necessary. Also, since $\left|B_{2 N / 3}^{y}\right| \leq N^{2}$ for any $y$,

$$
\left|D\left(\xi_{0}\right)\right|-\left|D\left(\xi_{\varepsilon_{2}}\right)\right|<\frac{\varepsilon^{\prime} R^{2}}{2}
$$

holds under $Z_{1}\left(\varepsilon_{2}\right)<\varepsilon^{\prime} m^{2} / 2$. So, $\left|D\left(\xi_{\varepsilon_{0}}\right)\right|>\varepsilon^{\prime} R^{2} / 2$ off of the exceptional set in (6.21). Together with (6.22), this implies (6.18).

We now apply Proposition 6.3, Lemma 6.9 and Proposition 6.16 to demonstrate Proposition 6.6. For this, we introduce the following notation. Set

$$
\begin{aligned}
S=\inf \{t: & K\left(\xi_{t}\right) \leq \bar{\varepsilon}_{1}\left(m^{2} \wedge N^{2}\right), \\
L\left(\xi_{t}\right) & \left.\leq \bar{\varepsilon}_{1} m^{2}, \xi_{t}(x) \neq 1 \text { at all } x \in B\right\},
\end{aligned}
$$

where, as before, $\bar{\varepsilon}_{1}$ denotes either $\varepsilon_{1}$ or $2 \varepsilon_{1}$. (Recall that $\varepsilon_{1}=1 / 8,000$.) Also, set

$$
\begin{aligned}
& F_{1}=\left\{\xi: K(\xi)=\left[\bar{\varepsilon}_{1}\left(m^{2} \wedge N^{2}\right)\right] \text { and } L(\xi) \leq\left\lfloor\bar{\varepsilon}_{1} m^{2}\right]\right\} \\
& F_{2}=\left\{\xi: K(\xi)<\left[\bar{\varepsilon}_{1}\left(m^{2} \wedge N^{2}\right)\right] \text { and } L(\xi)=\left[\bar{\varepsilon}_{1} m^{2}\right\rfloor\right\}
\end{aligned}
$$

If $B$ is occupied or well occupied under $\xi_{0}$, then

$$
\xi_{S} \in F_{1} \cup F_{2} \text { on } S<\infty .
$$

The stopping time $S$ occurs when $B$ borders on being unoccupied $\left(\bar{\varepsilon}_{1}=\varepsilon_{1}\right)$, or when $K$ and $L$ satisfy bounds that are twice as large $\left(\bar{\varepsilon}_{1}=2 \varepsilon_{1}\right)$. Since $K\left(\xi_{t}\right)$ and $L\left(\xi_{t}\right)$ can only decrease in increments of $1,(6.25)$ is easy to check. 
For $\xi_{S} \in F_{i}, i=1,2$, it follows from Lemma 6.9 that

$$
\left|D\left(\xi_{S}\right)\right| \geq \frac{R^{2}}{15} \geq \frac{R^{2}}{30} .
$$

If $\xi_{S} \in F_{1}$, we may therefore apply Proposition 6.3 , with $\varepsilon=1 / 30$ and $a=\left[\bar{\varepsilon}_{1}\left(m^{2} \wedge N^{2}\right)\right]$, together with the strong Markov property. If $\xi_{S} \in F_{2}$, we need to first apply (6.18) of Proposition 6.16, with $\varepsilon=\bar{\varepsilon}_{1}$ and $\varepsilon^{\prime}=1 / 15$, together with the strong Markov property, to obtain

$$
p\left(K\left(\xi_{S^{\prime}}\right) \leq \frac{N^{2}}{20} \text { or }\left|D\left(\xi_{S^{\prime}}\right)\right| \leq \frac{R^{2}}{30} \mid \mathscr{F}_{S}\right) \leq 3 \exp \left\{-\frac{C_{3} \bar{\varepsilon}_{1}}{15 \delta}\right\},
$$

where $S^{\prime}=S+\varepsilon_{2}$. On $G_{3}$, the complement of the exceptional set in (6.27), one may then apply Proposition 6.3, with $\varepsilon=1 / 30$ and $a=\left[\bar{\varepsilon}_{1}\left(m^{2} \wedge N^{2}\right)\right\rfloor$ as before. Set $S^{\prime \prime}$ equal to $S$ or $S^{\prime}$ as appropriate and $\sigma=S^{\prime \prime}+\varepsilon_{3}$, with $\varepsilon_{3}=1 /\left(3 \times 10^{6}\right)$. Using (6.4), one has in both cases that

$$
\begin{aligned}
& P\left(B \text { is poisoned by polymers at some } t \in\left[S^{\prime \prime}, \sigma\right] \mid \mathscr{F}_{S^{\prime \prime}}\right) \\
& \quad \leq C_{1} \exp \left\{-C_{4}\left(m^{2} \wedge N^{2}\right)\right\},
\end{aligned}
$$

for $\bar{\varepsilon}_{1}=\varepsilon_{1}$ and appropriate $C_{4}>0$, and

$$
\begin{aligned}
& P\left(K\left(\xi_{t}\right) \leq \varepsilon_{1}\left(m^{2} \wedge N^{2}\right) \text { for some } t \in\left[S^{\prime \prime}, \sigma\right] \mid \mathscr{F}_{S^{n}}\right) \\
& \quad \leq C_{1} \exp \left\{-C_{4}\left(m^{2} \wedge N^{2}\right)\right\}
\end{aligned}
$$

for $\bar{\varepsilon}_{1}=2 \varepsilon_{1}$. For both values of $\bar{\varepsilon}_{1},(6.5)$ implies that

$$
P\left(K\left(\xi_{\sigma}\right) \leq \bar{\varepsilon}_{1}\left(m^{2} \wedge N^{2}\right) \mid \mathscr{F}_{S^{\prime \prime}}\right) \leq C_{1} \exp \left\{-C_{4}\left(m^{2} \wedge N^{2}\right)\right\}
$$

Assume now that $B$ is initially occupied or well occupied. Employing the above results, we obtain the following behavior for $\xi_{t}$ over $[0, \sigma]$. On $[0, S)$, the square $B$ remains occupied. In particular, there are always either vacant sites or monomers in $B$. For $\xi_{S} \in F_{2}$, it follows from (6.17) of Proposition 6.16 (with the same choice of $\varepsilon$ and $\varepsilon^{\prime}$ ), that, off of an exceptional set, $B$ retains vacant sites on $\left[S, S^{\prime}\right)$, if $\bar{\varepsilon}_{1}=\varepsilon_{1}$; under $\bar{\varepsilon}_{1}=2 \varepsilon_{1}, B$ typically remains occupied. On [ $\left.S^{\prime \prime}, \sigma\right]$, it follows from (6.28) and (6.29), that, off of an exceptional set, $B$ again retains vacant sites under $\bar{\varepsilon}_{1}=\varepsilon_{1}$, whereas under $\bar{\varepsilon}_{1}=$ $2 \varepsilon_{1}, B$ remains occupied. Putting this together, we obtain the following proposition.

Proposition 6.31. Suppose that $m^{2} \wedge N^{2}$ is large relative to $1 / \delta$, with $\lambda \leq \delta m^{2}$. If $B$ is initially occupied, then for appropriate $C_{5}$ and $C_{6}>0$,

$$
P(B \text { is poisoned by polymers at some } t \in[0, \sigma]) \leq C_{5} \exp \left\{-\frac{C_{6}}{\delta}\right\}
$$

and

$$
P(B \text { is not occupied at } \sigma) \leq C_{5} \exp \left\{-\frac{C_{6}}{\delta}\right\}
$$


If $B$ is initially well occupied, then

$$
P(B \text { is not occupied at some } t \in[0, \sigma]) \leq C_{5} \exp \left\{-\frac{C_{6}}{\delta}\right\}
$$

and

$$
P(B \text { is not well occupied at } \sigma) \leq C_{5} \exp \left\{-\frac{C_{6}}{\delta}\right\} .
$$

(For $\sigma=\boldsymbol{S}=\infty$, we interpret $B$ as being well occupied under $\xi_{\infty}$.)

One is free to iterate the above procedure involving the behavior of $\xi_{t}$ over the interval $[0, \sigma]$. Setting $\sigma_{1}=\sigma$ and successively defining $\sigma_{j}$ in terms of $\sigma_{j-1}$, one can repeatedly employ Proposition 6.31 to analyze $\xi_{t}$ over $\left[0, \sigma_{j}\right]$. Recalling that $\sigma \geq \sigma-S^{\prime \prime}=\varepsilon_{3}$, one automatically has $\sigma_{j} \geq j \varepsilon_{3}$. Iterating over $t \in[0,2 / \delta]$, we therefore obtain the following bounds.

Proposition 6.36. Suppose that $m^{2} \wedge N^{2}$ is large relative to $1 / \delta$, with $\lambda \leq \delta m^{2}$. If $B$ is initially occupied, then for appropriate $C_{6}>0$ and $C_{7}$,

$P(B$ is poisoned by polymers at some $t \in[0,1 / \delta])$

$$
\leq \frac{C_{7}}{\delta} \exp \left\{-\frac{C_{6}}{\delta}\right\} \text {. }
$$

If $B$ is initially well occupied, then

$$
P(B \text { is not occupied at some } t \in[0,2 / \delta]) \leq \frac{C_{7}}{\delta} \exp \left\{-\frac{C_{6}}{\delta}\right\}
$$

Using Proposition 6.36, we are able to demonstrate Proposition 6.6 without difficulty. The bound (6.37) tells us that if a square $B(z)$, with $\|z\|=1$, is initially occupied, then it typically will not be poisoned by polymers for $T=1 / \delta$ units of time. Over this time interval, the neighbor $B=B(0)$ of $B(z)$ will typically be well occupied at some time $\tau$. The bound (6.38) then tells us that $B$ will typically remain occupied up to time $2 T$, which is the desired result.

Proof of Proposition 6.6. Every site in $B(0)$ is within range $R$ of every site in $B(z)$. So, if $B(z)$ contains a monomer at time $t, B(0)$ contains no polymer atoms then, and $B(0)$ will be well occupied. On the other hand, if $B(z)$ contains a vacant site $x$, then a polymer atom at a given site $y$ in $B(0)$ will desorb at least at rate $1 / 5 R^{2}$ due to monomers bonding at $x$. By Lemma 6.9 , as long as $B(0)$ is not well occupied, $\left|D\left(\xi_{t}\right)\right| \geq R^{2} / 15$. So, the rate at which polymer atoms desorb from $D\left(\xi_{t}\right)$ until $B(0)$ is well occupied at least $1 / 75$ as long as $B(z)$ remains unpoisoned by polymers.

Let $\tau$ denote the first time $B(0)$ is well occupied. It follows from (6.37) that, if $B(z)$ is initially occupied,

$$
P(\tau>T) \leq \frac{C_{7}}{\delta} \exp \left\{-\frac{C_{6}}{\delta}\right\}+\exp \left\{-\frac{1}{75 \delta}\right\} .
$$


If one applies the strong Markov property at time $\tau$, one obtains that over $[\tau, \tau+2 T], B(0)$ is occupied off of the exceptional set given in (6.38). So, if $B(z)$ is initially occupied,

$$
\begin{gathered}
P(B(0) \text { is not occupied at some } t \in[T, 2 T]) \\
\quad \leq \frac{2 C_{7}}{\delta} \exp \left\{-\frac{C_{6}}{\delta}\right\}+\exp \left\{-\frac{1}{75 \delta}\right\} .
\end{gathered}
$$

For sufficiently small $\delta>0$, it is clear that the right-hand side of (6.40) is dominated by $\delta$. This gives (6.7) and completes the proof of Proposition 6.6.

7. Demonstration of Proposition 6.3. In this section, we demonstrate Proposition 6.3. The basic idea behind the proof goes as follows. The assumption $\left|D\left(\xi_{0}\right)\right| \geq \varepsilon R^{2}$ says that at least a fixed proportion of the sites in $B^{I_{1}}$ are surrounded by polymer atoms. When a bonding monomer interacts with the polymer atom at such a site, $K\left(\xi_{t}\right)$ must increase on the order of magnitude of $N^{2}$ (Lemma 7.3). Moreover, as long as $D\left(\xi_{t}\right)$ remains large, a fixed fraction of the desorbing polymer atoms from $B^{I_{1}}$ will be from $D\left(\xi_{t}\right)$ (Corollary 7.7). One is therefore able to construct a supermartingale function of $K\left(\xi_{t}\right)$ for such $D\left(\xi_{t}\right)$ (Lemma 7.14). Typically, $D\left(\xi_{t}\right)$ will remain large for a fixed amount of time just depending on $\varepsilon$ (Corollary 7.23). Application of the optional sampling theorem and calculation of the exceptional probabilities in the above steps then yield the first part of Proposition 6.3. The same estimates, together with an elementary bound on the rate at which polymer atoms in $D\left(\xi_{t}\right)$ desorb (Lemma 7.28), yield the second part of Proposition 6.3. Reasons for the assumptions on $a$ in the statement of the proposition are given just before Lemma 7.25 and Lemma 7.28.

Although the above line of reasoning is in spirit correct, one needs to make several modifications for it to be executable. First of all, $D\left(\xi_{t}\right)$ is not exactly the right quantity to use, since we need to have more control over the prospective supermartingale. In particular, we are demanding too much in searching for sites $D\left(\xi_{t}\right)$ which are completely surrounded by polymer atoms. Such considerations also induce us to modify $K\left(\xi_{t}\right)$.

We begin by setting $\tilde{D}(\xi)$ equal to the set of those $x \in B^{I_{1}}$, with $\xi(x)=2$, for which the number of sites in $B_{2 N / 3}^{x}$ which are not occupied by polymer atoms is at most $N^{2} / 18$. It is easy to check that $D(\xi) \subset \tilde{D}(\xi)$ always holds. We define the quantities $A_{t}(\xi)$ and $\tilde{D}_{t}(\xi), t \geq 0$, inductively as follows. Choose $A_{0}(\xi) \in \mathscr{A}(\xi)$, where $\mathscr{A}(\xi)$ is defined in Section 6 , and set $\tilde{D}_{0}(\xi)$ equal to the set of those $y \in \tilde{D}(\xi)$ with $\|y-x\|_{\infty} \geq 2 N$ for all $x \in A_{0}(\xi)$. Employing the process $\xi_{t}$ with $\xi_{0}=\xi$, we extend $A_{t}(\xi)$ over time by adding a site $y \in \tilde{D}_{t-}(\xi)$ whenever a polymer atom desorbs at $y$, and removing a site $y$ whenever $\Gamma_{y}\left(\xi_{t}\right)=0$. Also, set $\tilde{D}_{t}(\xi)$ equal to those $y \in \tilde{D}\left(\xi_{t}\right)$ with $\|y-x\|_{\infty} \geq 2 N$ for all $x \in A_{t}(\xi)$. Using $A_{t}(\xi)$, we define

$$
K_{t}(\xi)=\sum_{x \in A_{t}(\xi)} \hat{\Gamma}_{x}\left(\xi_{t}\right)
$$


In this construction, one always has

$$
A_{t}\left(\xi_{0}\right) \in \mathscr{A}\left(\xi_{t}\right), \quad \tilde{D}_{t}\left(\xi_{0}\right) \subset \tilde{D}\left(\xi_{t}\right), \quad K_{t}\left(\xi_{0}\right) \leq K\left(\xi_{t}\right) .
$$

One can think of $\tilde{D}_{t-}(\xi)$ as providing the available pool of sites on which the "counter" $K_{t}(\xi)$ feeds when polymer atoms desorb. Typically, we will omit the argument $\xi_{0}$ and write $A_{t}=A_{t}\left(\xi_{0}\right), \tilde{D}_{t}=\tilde{D}_{t}\left(\xi_{0}\right)$ and $K_{t}=K_{t}\left(\xi_{0}\right)$.

In the above construction, when $y$ is added to $A_{t}, K_{t}$ increases by $\hat{\Gamma}_{y}\left(\xi_{t}\right)$. One has the following bounds, which are related to those in Lemma 6.13. Here and elsewhere throughout the section, none of the estimates depend on $\lambda$, the rate at which polymers bond. As in Section 6, we will also implicitly assume in this section that $m$ and $N$ are chosen large enough so that certain nuisance constants can be absorbed into the bounds by an appropriate change in factor.

LEMMA 7.3. Suppose that $y \in \tilde{D}_{t-}$ and $\xi_{t}(y)=0$. Then, $\hat{\Gamma}_{y}\left(\xi_{t}\right) \geq N^{2} / 20$, and hence

$$
K_{t}-K_{t-} \geq N^{2} / 20 \text {. }
$$

Proof. Any $N \times N$ square containing $y$, when intersected with $B_{2 N / 3}^{y}$, contains a square $B_{N / 3}^{z}$. Since $y \in \tilde{D}_{t-} \subset \tilde{D}\left(\xi_{t-}\right), B_{N / 3}^{z}$ has at most $N^{2} / 18$ vacant sites at time $t-$. For large $N,\left|B_{N / 3}^{z}\right| \approx N^{2} / 9$. So, at least $N^{2} / 20$ polymer atoms must be removed after time $t$ in order for a polymer to be able to bond on a square containing $y$. Hence, $\hat{\Gamma}_{y}\left(\xi_{t}\right) \geq N^{2} / 20$. Since $y \notin A_{t-}$ and $y \in A_{t}$, this implies (7.4).

Recall that $\bar{B}=B_{M+2 R}^{0}$ is the set of all sites within distance $R$ of $B$, and that $|\bar{B}| \leq 7 R^{2}$. Since $\left.M=2 \mid R / 4\right\rfloor$, it is not hard to check that for each $x \in \bar{B}$, there are at least $R^{2} / 4$ sites $y$ with $\|x-y\|_{\infty} \leq R$ and $\|y-z\|_{\infty} \leq R$ for every $z \in B$. For any $\xi$, it is therefore either the case that (a) for any $x \in \bar{B}$, the number of sites $y$ with $\|x-y\|_{\infty} \leq R$ and $\xi(y)=2$ is at least $R^{2} / 8$ or (b) the number of sites $y$ with $\|y-z\|_{\infty} \leq R$ for every $z \in B$ and $\xi(y) \neq 2$ is at least $R^{2} / 8$. As we shall see, these conditions say in essence that either (a) no polymer atom present in $B$ desorbs at a fast rate or (b) all polymer atoms in $B$ desorb at at least a moderate rate. This alternative is employed in Lemma 7.5 below. Here and later on, we use the following terminology. We distinguish between polymer atoms that desorb immediately upon bonding on $\mathbf{Z}^{2}$ through interaction with an already present monomer, and those polymer atoms that remain on $\mathbf{Z}^{2}$ for a positive period of time and desorb only when a monomer bonds. We refer to the first type as instantaneous polymer atoms and the second type as noninstantaneous polymer atoms. We set $r_{A}(\xi)=$ the rate at which any noninstantaneous polymer atoms desorbs from the set $A \subset \mathbf{Z}^{2}$ for the configuration $\xi$, and set $P_{A}(\xi)=$ the number of polymer atoms in $A$. Denote by $\mathscr{A}^{\prime}(\xi)$ the collection of sets $A \in \mathscr{A}(\xi)$, where, in addition, for $x \in A$, the square $B_{2 N}^{x}$ contains at least $N^{2} / 2$ vacant sites, and, by $U(A)=\cup_{x \in A} B_{2 N}^{x}$, the union of the squares. Note that $B_{2 N}^{x}$ contains any polymer which contains $x$, and that $B_{2 N}^{x_{1}} \cap B_{2 N}^{x_{2}}$ $=\varnothing$ for $x_{1} \neq x_{2}$ and $x_{1}, x_{2} \in A \in \mathscr{A}^{\prime}(\xi)$. 
LEMMA 7.5. Assume that $A \subset B$, with $P_{A}(\xi) \geq \varepsilon R^{2}$ for some $\varepsilon>0$. Then, for any $A^{\prime} \in \mathscr{A}^{\prime}(\xi)$ with $A^{\prime} \neq \varnothing$,

$$
\frac{r_{A}(\xi)}{r_{U_{\left(A^{\prime}\right)}}(\xi)} \geq \frac{\varepsilon}{2,500}
$$

Note that on $A^{\prime}=\varnothing, r_{U\left(A^{\prime}\right)}(\xi)$ is trivially 0 .

Let $A_{t}^{\prime}$ denote the subset of $A_{t}$ consisting of those $x$ for which $B_{2 N}^{x}$ contains at least $N^{2} / 2$ vacant sites. Also, let

$$
\tau_{1}=\tau_{1}^{\varepsilon}=\inf \left\{t:\left|\tilde{D}_{t}\right| \leq \frac{\varepsilon}{4} R^{2}\right\}
$$

for $\varepsilon>0$. (Here and elsewhere in the section, we suppress the superscript for stopping times $\tau_{i}^{\varepsilon}$ depending on $\varepsilon$.) Then, the following is an immediate consequence of Lemma 7.5.

COROLlaRY 7.7. Assume that $t<\tau_{1}$ for a given $\varepsilon>0$ and that $A_{t}^{\prime} \neq \varnothing$. Then,

$$
\frac{r_{\bar{D}_{t}}\left(\xi_{t}\right)}{r_{U\left(A_{t}^{\prime}\right)}\left(\xi_{t}\right)} \geq \frac{\varepsilon}{10,000}
$$

Proof of Lemma 7.5. Assume first, as in (b) above, that the number of vacant sites within range $R$ of every site in $B$ is at least $R^{2} / 8$. One trivially has

$$
r_{U\left(A^{\prime}\right)}(\xi) \leq r_{B}(\xi) \leq 7 R^{2},
$$

since the number of sites in $\bar{B}$ is less than $7 R^{2}$. On the other hand, since $P_{A}(\xi) \geq \varepsilon R^{2}$ and the number of sites a given monomer can interact with is at most $5 R^{2}$,

$$
r_{A}(\xi) \geq \frac{R^{2}}{8} \frac{\varepsilon R^{2}}{5 R^{2}} \geq \frac{\varepsilon}{40} R^{2}
$$

Taking the ratio of the bounds in (7.9) and (7.10), we see that (7.6) holds under (b).

Suppose now, as in (a), that for any $x \in \bar{B}$, the number of sites $y$ within range $R$ of $x$ with $\xi(y)=2$ is at least $R^{2} / 8$. Since

$$
P_{U\left(A^{\prime}\right)}(\xi) \leq 4\left|A^{\prime}\right| N^{2}
$$

trivially holds, the fraction of polymer atoms within range $R$ of $x$ which are contained in $U\left(A^{\prime}\right)$ is at most $32\left|A^{\prime}\right| N^{2} / R^{2}$. It follows that

$$
r_{U\left(A^{\prime}\right)}(\xi) \leq 7 R^{2} \frac{32\left|A^{\prime}\right| N^{2}}{R^{2}}=224\left|A^{\prime}\right| N^{2} .
$$


On the other hand, since $U\left(A^{\prime}\right)$ contains at least $\left|A^{\prime}\right| N^{2} / 2$ vacant sites, it also follows that

$$
r_{A}(\xi) \geq \frac{\left|A^{\prime}\right| N^{2}}{2} \frac{\varepsilon R^{2}}{5 R^{2}}=\frac{\varepsilon}{10}\left|A^{\prime}\right| N^{2} .
$$

Taking the ratio of (7.11) and (7.12), we see that (7.6) holds under (a) as well.

By employing Lemma 7.3 and Corollary 7.7 , one can show that $K_{t}$ tends to increase for $t \leq \tau_{1}$. To make this precise, we introduce the function $Y_{t}$, $t \in[0,1]$ with

$$
Y_{t}=Y_{t}^{\varepsilon, \beta}= \begin{cases}\exp \left\{-\beta K_{t}\right\}, & \text { for } t \in\left[0, \tau_{1}\right] \\ \exp \left\{-\beta K_{\tau_{1}}\right\}, & \text { for } t \in\left[\tau_{1}, 1\right]\end{cases}
$$

where $\varepsilon>0$ and $\beta>0$. (The finite bound $t \leq 1$ is not necessary, but shortens the proof of Lemma 7.14 by a step.) Also, let

$$
\tau_{2}(t)=\inf \left\{u>t: K_{u}-K_{u-}=-1 \text { or } K_{u}-K_{u-} \geq N^{2} / 20\right\} \wedge \tau_{1} \wedge 1 .
$$

Lemma 7.14 shows that $Y_{t}$ is a supermartingale. Here, the choice of $\hat{\Gamma}_{x}$ rather than $\Gamma_{x}$ in the definition of $K_{t}$ in (7.1) is important, since one can control the ratio in (7.8).

LEMMA 7.14. Assume that $1 / N^{2}$ and $\beta$ are small relative to $\varepsilon$. Then, $Y_{t}^{\varepsilon, \beta}$ is a supermartingale with respect to $\mathscr{F}_{t}$ on $t \leq 1$.

Proof. Note that $K_{t}$ can only decrease when a polymer atom is removed from $U\left(A_{t_{-}}^{\prime}\right)$, in which case it decreases by 1 . By Lemma 7.3 , on the other hand, $K_{t}$ increases by at least $N^{2} / 20$ when a polymer atom is removed from $\tilde{D}_{t-}$. Also, by Corollary 7.7 ,

$$
\frac{r_{\tilde{D}_{t}}\left(\xi_{t}\right)}{r_{U\left(A_{t}^{\prime}\right)}\left(\xi_{t}\right)} \geq \frac{\varepsilon}{10,000}
$$

for $t<\tau_{1}$ and $A_{t}^{\prime} \neq \varnothing$. Set $\bar{\varepsilon}=\varepsilon / 10,000$. Assuming $t<\tau_{1}$, one therefore has

$$
P\left(K_{\tau_{2}(t)}-K_{t} \geq \frac{N^{2}}{20} \mid \mathscr{F}_{t}\right) \geq \bar{\varepsilon} P\left(K_{\tau_{2}(t)}-K_{t}=-1 \mid \mathscr{F}_{t}\right),
$$

with $K_{\tau_{2}(t)} \geq K_{t}$ off of these two sets.

Using (7.15), we show that

$$
E\left[Y_{\tau_{2}(t)} \mid \mathscr{F}_{t}\right] \leq Y_{t}
$$

for large $N$ and small $\beta$. Since $K_{u}$ is increasing over $\left[t, \tau_{2}(t)\right.$ ), a little thought shows that $Y_{t}$ is therefore a supermartingale. To obtain (7.16), we set

$$
\rho_{t}=P\left(K_{\tau_{2}(t)}-K_{t}=-1 \mid \mathscr{F}_{t}\right) \text {. }
$$


It then follows from (7.15) that

$$
\begin{aligned}
& E\left[Y_{\tau_{2}(t)} \mid \mathscr{F}_{t}\right]-Y_{t} \\
& \quad \leq \rho_{t}\left[\exp \left\{-\beta\left(K_{t}-1\right)\right\}-\exp \left\{-\beta K_{t}\right\}\right] \\
& \quad+\bar{\varepsilon} \rho_{t}\left[\exp \left\{-\beta\left(K_{t}+\frac{N^{2}}{20}\right)\right\}-\exp \left\{-\beta K_{t}\right\}\right] \\
& \quad=\rho_{t} \exp \left\{-\beta K_{t}\right\}\left[(\exp \{\beta\}-1)+\bar{\varepsilon}\left(\exp \left\{-\frac{\beta N^{2}}{20}\right\}-1\right)\right] .
\end{aligned}
$$

By expanding out the exponentials in [-] above, it is easy to check that for $N^{2} \geq 40 / \bar{\varepsilon}$ and $\beta$ a small enough multiple of $\varepsilon$, the quantity in [.] is at most 0 . This gives (7.16). Consequently, $Y_{t}$ is a supermartingale. (Note that, along the lines of the discussion at the end of Section 4 on the coupling of different systems, the corresponding supermartingales $Y_{t}$ can be coupled by matching the downward and upward jumps in each case.)

In Lemma 7.17 , we bound $\tilde{D}\left(\xi_{t}\right)$ in terms of $D\left(\xi_{0}\right)$ by controlling the number of monomers that can bond in $\bar{B}$ up to time $t$. This bound is much better than any analog involving $D\left(\xi_{t}\right)$, and is the reason for introducing $\tilde{D}\left(\xi_{t}\right)$. As in Section 6 , the term $V_{A}(\xi)$ denotes the number of vacant sites in $A$.

LemMa 7.17. Assume that $\left|D\left(\xi_{0}\right)\right| \geq \varepsilon R^{2}$ for some $\varepsilon>0$. Then,

$$
P\left(\left|\tilde{D}\left(\xi_{t}\right)\right| \leq \frac{\varepsilon R^{2}}{2} \text { at some } t \leq \frac{\varepsilon}{1,100}\right) \leq \exp \left\{-\frac{c_{4} \varepsilon R^{2}}{144}\right\},
$$

where $c_{4}>0$ is as in (5.10).

Proof. Suppose $x \in D\left(\xi_{0}\right)$. For $x \notin \tilde{D}\left(\xi_{t}\right)$, one has either

$$
\xi_{t}(x) \neq 2 \text { or } V_{B_{2 N / 3}^{x}}\left(\xi_{i}\right)>\frac{N^{2}}{18}
$$

For $\left|D\left(\xi_{0}\right)\right|-\left|\tilde{D}\left(\xi_{t}\right)\right| \geq \varepsilon R^{2} / 2$ to hold, at least $\varepsilon R^{2} / 4$ sites $x \in D\left(\xi_{0}\right)$ need to satisfy either the first half or the second half of (7.19). In the first case, one has, of course,

$$
\left|\left\{x \in B^{I_{1}}: \xi_{0}(x)=2, \xi_{t}(x) \neq 2\right\}\right| \geq \frac{\varepsilon R^{2}}{4} .
$$

For any $x \in D\left(\xi_{0}\right)$ and $y \in B_{2 N / 3}^{x}$, one has $\xi_{0}(y)=2$. So, under the second half of (7.19), more than $N^{2} / 18$ sites in $B^{I_{1}}$ have become vacant over $[0, t]$. Also, for any $x,\left|B_{2 N / 3}^{x}\right| \leq\left(\frac{2}{3} N+1\right)^{2} \leq N^{2}$. It therefore follows in the second case, that

$$
\left|\left\{x \in B^{I_{1}}: \xi_{0}(x)=2, \xi_{t}(x) \neq 2\right\}\right| \geq \frac{N^{2}}{18} \frac{\varepsilon R^{2}}{4} \frac{1}{N^{2}}=\frac{\varepsilon R^{2}}{72} .
$$

So, in either case, the quantity on the left-hand side is at least $\varepsilon R^{2} / 72$. 
Each time a noninstantaneous polymer atom desorbs from $B^{I_{1}}$, a monomer atom needs to bond in $\bar{B}$. The number of such monomers bonding over $[0, u]$ is trivially dominated by a Poisson random variable $X$ with mean $7 R^{2} u$. It therefore follows from (5.10) with $\eta=1$ and $u=\varepsilon / 1,100<\varepsilon /(14 \cdot 72)$, and (7.20) and (7.21), that

$$
\begin{aligned}
P\left(\left|D\left(\xi_{0}\right)\right|-\left|\tilde{D}\left(\xi_{t}\right)\right| \geq \frac{\varepsilon R^{2}}{2} \text { at some } t \leq \frac{\varepsilon}{1,100}\right) & \leq P\left(X \geq \frac{\varepsilon R^{2}}{72}\right) \\
& \leq \exp \left\{-\frac{c_{4} \varepsilon R^{2}}{144}\right\} .
\end{aligned}
$$

This implies (7.18).

We introduce the stopping time

$$
\tau_{3}=\inf \left\{t:\left|A_{t}\right|>\frac{\varepsilon m^{2}}{64}-1\right\} .
$$

Using $\tau_{3}$, we can reinterpret Lemma 7.17 in terms of $\tilde{D}_{t}$.

CoRollaRY 7.23. Assume that $\left|D\left(\xi_{0}\right)\right| \geq \varepsilon R^{2}$ for some $\varepsilon>0$. Then

$$
\begin{aligned}
P\left(\tau_{1} \leq \frac{\varepsilon}{1,100} \wedge \tau_{3}\right) & =P\left(\left|\tilde{D}_{t}\right| \leq \frac{\varepsilon}{4} R^{2} \text { at some } t \leq \frac{\varepsilon}{1,100} \wedge \tau_{3}\right) \\
& \leq \exp \left\{-\frac{c_{4} \varepsilon R^{2}}{144}\right\}
\end{aligned}
$$

PRoof. When $\left|A_{t}\right| \leq \varepsilon m^{2} / 64$, there are at most $\left(\varepsilon m^{2} / 64\right)\left(16 N^{2}\right)=$ $\varepsilon R^{2} / 4$ sites strictly within distance $2 N$ of any $x \in A_{t}$. These are the only points that are not in $\tilde{D}_{t}$, but might be in $\tilde{D}\left(\xi_{t}\right)$. On $t \leq \tau_{3},\left|A_{t}\right| \leq \varepsilon \mathrm{m}^{2} / 64$, and therefore

$$
\left|\tilde{D}\left(\xi_{t}\right)\right|-\left|\tilde{D}_{t}\right| \leq \frac{\varepsilon R^{2}}{4} .
$$

The inequality therefore follows from (7.18). The equality follows from the definition of $\tau_{1}$.

In order to be able to apply Corollary 7.23 , we also need to be able to rule out the possibility that $K_{t}$ behaves badly after time $\tau_{3}$. This is done in Lemma 7.25. The condition $\left|A_{0}\right| \leq \varepsilon \mathrm{m}^{2} / 128$ here is the reason for the assumption $a \leq \varepsilon \mathrm{m}^{2} / 128$ in the statement of Proposition 6.3. The absence of a suitable analog for $K\left(\xi_{t}\right)$ is the reason for introducing $K_{t}$.

LEMMA 7.25. For given $\varepsilon>0$, assume that $m$ is large relative to $1 / \varepsilon$, and that $\left|A_{0}\right| \leq \varepsilon m^{2} / 128$. Then,

$$
P\left(K_{t} \leq \frac{\varepsilon R^{2}}{10^{4}} \text { at some } t \in\left(\tau_{3}, \frac{\varepsilon}{10^{5}}\right]\right) \leq \exp \left\{-\frac{c_{4} \varepsilon R^{2}}{10^{5}}\right\} \text {. }
$$


Proof. Since $\left|A_{0}\right| \leq \varepsilon m^{2} / 128$ and $\left|A_{\tau_{3}}\right|>\varepsilon m^{2} / 64-1$, one has

$$
\left|A_{\tau_{3}}\right|-\left|A_{0}\right|>\frac{\varepsilon m^{2}}{128}-1 \text {. }
$$

This implies that there are sites $y_{1}, \ldots, y_{i_{0}}$, with $\left\|y_{i}-y_{i} \cdot\right\|_{\infty} \geq 2 N$ and $i_{0}=$ $\left[\varepsilon m^{2} / 128-1\right]$, where at certain times $\alpha_{i} \leq \tau_{3}$,

$$
y_{i} \in \tilde{\mathscr{D}}_{\alpha_{i}-} \text { and } \xi_{\alpha_{i}}\left(y_{i}\right)=0 .
$$

So, $y_{i} \in A_{\alpha_{i}}$, and by Lemma 7.3 ,

$$
\hat{\Gamma}_{y_{i}}\left(\xi_{\alpha_{i}}\right) \geq \frac{N^{2}}{20}
$$

It follows, for $t \geq \tau_{3}$, that at least $i_{0} N^{2} / 40$ noninstantaneous polymer atoms need to desorb on $B$ over $[0, t]$ in order for $K_{t} \leq i_{0} N^{2} / 40$. For $m$ large relative to $1 / \varepsilon, i_{0} N^{2} / 40 \geq \varepsilon R^{2} / 10^{4}$. On the other hand, the number of noninstantaneous polymer atoms which desorb from $B$ over $[0, u]$ is dominated by a Poisson random variable $X$ with mean $7 R^{2} u$. So, by (5.10), with $\eta=2 / 5$ and $u=\varepsilon / 10^{5}$,

$$
P\left(K_{t} \leq \frac{\varepsilon R^{2}}{10^{4}} \text { at some } t \in\left(\tau_{3}, \frac{\varepsilon}{10^{5}}\right]\right) \leq P\left(X \geq \frac{\varepsilon R^{2}}{10^{4}}\right) \leq \exp \left\{-\frac{c_{4} \varepsilon R^{2}}{10^{5}}\right\} \text {. }
$$

In the demonstration of (6.5), we will use Lemma 7.28. It employs the observation that when $\tilde{D}_{t}$ is large and $K_{t}$ is small, the rate at which polymer atoms desorb from $\tilde{D}_{t}$ is high. Such a desorption then increases $K_{t}$. The need to always have on the order of $N^{2}$ vacant sites in $B$ in the proof of the lemma is the reason for the assumption bounding $a$ in terms of $N^{2}$ in the statement of Proposition 6.3.

LEMMA 7.28. For given $\varepsilon>0$, and $m$ large relative to $1 / \varepsilon$,

$$
P\left(\tilde{D}_{t} \geq \frac{\varepsilon}{4} R^{2} \text { and } 0<K_{t}<\frac{N^{2}}{20} \text { at all } t \leq \frac{\varepsilon}{10^{5}}\right) \leq \exp \left\{-\frac{\varepsilon N^{2}}{10^{7}}\right\}
$$

Proof. Under $0<K_{t}<N^{2} / 20$, there must be an $N \times N$ square contained in $B$ with more than $19 N^{2} / 20$ vacant sites. When $\left|\tilde{D}_{t}\right| \geq R^{2} / 10$, this implies that the rate at which polymer atoms desorb from $\tilde{D}_{t}$ is at least $\left(19 N^{2} / 20\right)\left(R^{2} / 10\right)\left(1 / 5 R^{2}\right) \geq N^{2} / 100$. For large $m$, there are more than $R^{2} / 5$ sites in $B^{I_{1}}$. So, when $\left|\tilde{D}_{t}\right|<R^{2} / 10$, either

$$
\xi_{t}(x) \neq 2 \text { or } \quad V_{B_{2 N / 3}^{x} / 3}\left(\xi_{t}\right)>\frac{N^{2}}{18}
$$

holds for at least $R^{2} / 20$ sites in $x \in B^{I_{1}} \cap \tilde{D}_{t}^{c}$. Reasoning as in (7.20) and (7.21), one can show that in either case, the number of vacant sites in $B$ is at least $R^{2} / 360$. So, when $\left|\tilde{D}_{t}\right| \in\left[\varepsilon R^{2} / 4, R^{2} / 10\right)$, the rate at which polymers 
desorb is at least $\left(R^{2} / 360\right)\left(\varepsilon R^{2} / 4\right)\left(1 / 5 R^{2}\right)=\varepsilon R^{2} / 7,200$; for $m$ large relative to $1 / \varepsilon$, this is again at least $N^{2} / 100$. The probability that this rate holds over $\left[0, \varepsilon / 10^{5}\right]$ without any polymer atom in $\tilde{D}_{t}$ desorbing is at most $\exp \left\{-\varepsilon N^{2} / 10^{7}\right\}$. If such a polymer atom desorbs at time $t$, then $K_{t} \geq N^{2} / 20$ by Lemma 7.3. This implies (7.29). (Note that, along the lines of the discussion at the end of Section 4 on the coupling of different systems, the above bounds do not depend on the precise configuration of $\xi_{t}$, but rather on the number of vacant sites and the size of $\tilde{D}_{t}$. So, the corresponding exceptional sets may be coupled.)

We now demonstrate Proposition 6.3. The proof of (6.4) consists of applying the optional sampling theorem to the supermartingale $Y_{t}$ from Lemma 7.14 and using the estimates for the exceptional sets given in Corollary 7.23 and Lemma 7.25. The proof of (6.5) consists of analogous estimates, together with Lemma 7.28.

Proof of Proposttion 6.3. One can choose $A_{0} \in \mathscr{A}\left(\xi_{0}\right)$ so that $\left|A_{0}\right| \leq$ $\varepsilon m^{2} / 128$ and $K_{0} \geq a$, since $\Gamma_{x}\left(\xi_{0}\right) \geq 1$ for each $x \in A_{0}$. For the process $K_{t}$ thus defined, it follows from Lemma 7.14 that $Y_{t}$ is a positive supermartingale for small enough $\beta>0$. Set

$$
\tau_{4}=\inf \left\{t: K_{t} \leq \frac{a}{2}\right\} \wedge \tau_{1} .
$$

By the optional sampling theorem,

$$
E\left[Y_{\tau_{4}}\right] \leq E\left[Y_{0}\right] \leq \exp \{-\beta a\} .
$$

It therefore follows from Chebyshev's inequality that

$$
P\left(K_{t} \leq \frac{a}{2} \text { at some } t \leq \tau_{1}\right) \leq \exp \left\{-\frac{\beta a}{2}\right\} .
$$

On the other hand, by Corollary 7.23 ,

$$
P\left(\tau_{1} \leq \varepsilon^{\prime} \wedge \tau_{3}\right) \leq \exp \left\{-\frac{c_{4} \varepsilon R^{2}}{144}\right\}
$$

since $\left|D\left(\xi_{0}\right)\right| \geq \varepsilon R^{2}$, where we abbreviate $\varepsilon^{\prime}=\varepsilon / 10^{5}$. Also, by Lemma 7.25 ,

$$
P\left(K_{t} \leq \frac{a}{2} \text { at some } t \in\left(\tau_{3}, \varepsilon^{\prime}\right]\right) \leq \exp \left\{-\frac{c_{4} a}{20}\right\},
$$

for large enough $m$ or $N$, since $\left|A_{0}\right| \leq \varepsilon m^{2} / 128$ and $a \leq \varepsilon\left(m^{2} \wedge N^{2}\right) / 128$. Putting (7.33)-(7.35) together, it follows that

$$
P\left(K_{t} \leq \frac{a}{2} \text { at some } t \leq \varepsilon^{\prime}\right) \leq C_{1} \exp \left\{-C_{2} a\right\}
$$

for appropriate $C_{1}$ and $C_{2}>0$. Since $K_{t} \leq K\left(\xi_{t}\right)$ always holds, this implies (6.4). 
To show (6.5), we first note that at least one of the following five events must occur: (1) $\tau_{3}<\varepsilon^{\prime}$, (2) $\tau_{3} \geq \varepsilon^{\prime}$, but $\tau_{1} \leq \varepsilon^{\prime}$ (i.e., $\tilde{D}_{t}<(\varepsilon / 4) R^{2}$ for some $\left.t \leq \varepsilon^{\prime}\right)$, (3) $K_{t}=0$ for some $t \leq \varepsilon^{\prime}$, (4) $\tilde{D}_{t} \geq(\varepsilon / 4) R^{2}$ and $0<K_{t}<N^{2} / 20$ for all $t \leq \varepsilon^{\prime}$, or (5) $\tilde{D}_{t} \geq(\varepsilon / 4) R^{2}$ for all $t \leq \varepsilon^{\prime}$ and $K_{t} \geq N^{2} / 20$ for some $t \leq \varepsilon^{\prime}$. Denote these events by $E_{1}, \ldots, E_{5}$. One can bound $P\left(K\left(\xi_{\varepsilon^{\prime}}\right) \leq 2 a ; E_{i}\right)$ for all $i$ by using previous estimates. By Lemma 7.25, for large enough $m$ or $N$,

$$
P\left(K_{\varepsilon^{\prime}} \leq 2 a ; E_{1}\right) \leq \exp \left\{-\frac{c_{4} a}{5}\right\} .
$$

The bound for $P\left(E_{2}\right)$ is given in (7.34), and that for $P\left(E_{3}\right)$ is given in (7.36). By Lemma 7.28,

$$
P\left(E_{4}\right) \leq \exp \left\{-\frac{\varepsilon N^{2}}{10^{7}}\right\} .
$$

In order to obtain the behavior under $E_{5}$, we translate the process in time by

and set

$$
\tau_{5}=\inf \left\{t: K_{t} \geq \frac{N^{2}}{20}\right\},
$$

$$
\bar{K}_{t}=K_{t+\tau_{5}} \text { and } \bar{Y}_{t}=Y_{t+\tau_{5}} .
$$

One can apply the same reasoning as in (7.31)-(7.33) to $\bar{K}_{t}$ and $\bar{Y}_{t}$, but with $4 a$ replacing $a$ (the estimates are valid for any fixed multiple of $a$ ). Since $4 a \leq N^{2} / 20$, it follows, in particular, that

$$
P\left(K_{\varepsilon^{\prime}} \leq 2 a ; E_{5}\right) \leq \exp \{-2 \beta a\} .
$$

Combining the inequalities between (7.37) and (7.39), for $E_{1}, \ldots, E_{5}$, shows that

$$
P\left(K_{\varepsilon^{\prime}} \leq 2 a\right) \leq C_{1} \exp \left\{-C_{2} a\right\}
$$

for appropriate $C_{1}, C_{2}>0$. Again, since $K_{t} \leq K\left(\xi_{t}\right)$ always holds, this implies (6.5). (Note that if one wishes to couple different systems along the lines of the discussion at the end of Section 4 , then the coupling needs to be restarted at time $\tau_{5}$.)

8. Monomer survival for $\boldsymbol{\lambda}>\boldsymbol{\delta} \mathbf{m}^{2}$. Basic setup. In Sections $\mathbf{6}$ and 7 , we showed that monomers survive for a long range of interaction and large polymer size, if the rate at which polymers bond, $\lambda$, satisfies $\lambda \leq \delta m^{2}$ for appropriate $\delta>0$. The main result employed there, Proposition 6.6, stated that for $T=1 / \delta$ and some $B(z),\|z\|_{1}=1$, occupied at time $0, B(0)$ is occupied over [T, 2T] except on a set of probability $\delta$. Proposition 6.3, Lemma 6.9 and Proposition 6.16 were the main tools used in demonstrating Proposition 6.6, with the demonstration of Proposition 6.3 occupying Section 7.

The argument for $\lambda>\delta m^{2}$ follows along the same lines, but involves certain complications. Proposition 6.3 does not depend on $\lambda$, but Lemma 6.9 and Proposition 6.16 need to be rephrased to be applicable here. In particular, 
the lower bounds in (6.17) and (6.18) of Proposition 6.16 depended on the restriction, $\lambda \leq \delta \mathrm{m}^{2}$, given in Lemma 6.14. The basic problem is that if one has no control over the rate at which polymers bond, then large vacant regions in the $M \times M$ square $B$ can conceivably be poisoned by polymers before bonding monomers have a chance to poke holes in solid polymer regions, which was the strategy employed in Section 6 .

In this section, we address this problem. If one restricts oneself to vacant regions where polymers do not bond too rapidly, then the analog of Proposition 6.16, Proposition 8.3, holds. To state things properly there and later on, we first need to introduce some terminology involving "slow" and "quick" $N \times N$ squares. In particular, the definition of "occupied" will be somewhat different here than in Section 6 and employs these ideas. With these changes, the main result in this section, Proposition 8.6, is analogous to Proposition 6.6. The basic reasoning leading to Proposition 6.6, in (6.23) through (6.40), is repeated here [(8.9)-(8.55)], although there are certain complications. Instead of the two cases $F_{1}$ and $F_{2}$, there are now four cases to consider [(8.12)]. Also, more work is required to analyze the behavior of $\xi_{t}$ on $[0, \hat{S}]$, the analog of $\left[0, S^{\prime \prime}\right]$ in Section 6 , in two of the cases. Because of the related structure of the two sections, we attempt to limit ourselves as much as possible to explaining the new arguments present in this section, while keeping the comparisons with Section 6 intelligible.

In Proposition 6.16, the presence of a large number $L$ of vacant $N \times N$ squares was required. These squares also entered into the definition of occupied for $\lambda \leq \delta m^{2}$. Here, we divide such squares into two types, "slow" squares and "quick" squares. As in Section 6 , for any configuration $\xi$ with $\xi(x) \neq 1$ for all $x \in B$, let $\mathscr{P}(\xi)=\left\{P_{1}(\xi), \ldots, P_{L}(\xi)\right\}$ denote a collection of vacant $N \times N$ squares $B_{N}^{y_{3}}, l=1, \ldots, L$, satisfying $\left\|y_{l_{1}}-y_{l_{2}}\right\|_{\infty} \geq 7 N$ and $B_{N}^{y_{2}} \subset B^{I_{3}}$. We say that a vacant square $B_{N}^{y}$ is slow for the configuration $\xi$ if the rate of bonding polymers which intersect $B_{N}^{y}$ is at most $\mu=4 \delta R^{2}$, for fixed $\delta>0$; we write $P_{l}(\xi) \in \mathscr{P}(\xi)$, if $P_{l}(\xi) \in \mathscr{P}(\xi)$ is slow. Similarly, we say that $B_{N}^{y}$ is quick if the rate is greater than $4 \delta R^{2}$, and that $P_{l}(\xi) \in \mathscr{Q}(\xi)$, if $P_{l}(\xi) \in \mathscr{P}(\xi)$ is quick. As just before Lemma 6.14, $\lambda \leq \delta m^{2}$ automatically implies this rate is at most $4 \delta R^{2}$, and so the vacant squares dealt with in Section 6 are all slow. Here, we choose $\mathscr{P}(\xi)$ by maximizing $|\mathscr{S}(\xi)|$ first, and then by maximizing $|\mathscr{P}(\xi)|$ among the collections satisfying this condition on $|\mathscr{S}(\xi)|$.

We also employ certain subsets $\mathscr{S}_{t}(\xi)$ and $\mathscr{H}_{t}(\xi), t \geq 0$, of $\mathscr{P}(\xi)$. For $P_{l}(\xi) \in \mathscr{S}(\xi)$, let $P_{l}(\xi) \in \mathscr{S}_{t}(\xi)$ if no polymer intersecting with $P_{l}(\xi)$ has bonded by time $t$ and no polymer atom in $P_{l}^{3}(\xi)$ (defined above Lemma 6.9) has desorbed by then. Clearly, $\mathscr{S}_{t}(\xi) \subset \mathscr{A}(\xi)$ and any $P_{l}(\xi) \in \mathscr{S}_{t}(\xi)$ is still vacant. Since the rate at which bonding polymers intersect $P_{l}(\xi) \in \mathscr{S}_{t}(\xi)$ is still at most $\mu$ at time $t$,

$$
\left|\mathscr{S}_{t}(\xi)\right| \leq\left|\mathscr{S}\left(\xi_{t}\right)\right|
$$

as well. For $P_{l}(\xi) \in \mathscr{Q}(\xi)$, let $P_{l}(\xi) \in \mathscr{F}_{t}(\xi)$ if a polymer intersecting $P_{l}^{3}(\xi)$ has bonded by time $t$. One can check that until $P_{l}(\xi)$ enters $\mathscr{F}_{t}(\xi)$, the bonding rate for polymers intersecting $P_{l}(\xi)$ is greater than $\mu$. 
Using this terminology, we now tailor Lemma 6.9 and Proposition 6.16 to our needs. In Lemma 8.1, we substitute $|\mathscr{S}(\xi)|+|\mathscr{Q}(\xi)|$ for $L(\xi)$, and in Proposition 8.3, $\left|\mathscr{S}\left(\xi_{0}\right)\right|$ for $L\left(\xi_{0}\right)$. One can check that the exact same reasoning as in the proof of Lemma 6.9 works for Lemma 8.1. For Proposition 8.3, a quick extra step needs to be added to the proof of Proposition 6.16 because of the desorption of polymer atoms in $P_{l}^{3}(\xi)$, but the rest of the reasoning is the same. We omit the arguments here.

LemMa 8.1. Assume that $B$ contains no monomers for a given configuration $\xi$, and that $K(\xi) \leq m^{2} / 400,|\mathscr{P}(\xi)| \leq m^{2} / 8,000$ and $|\mathscr{Q}(\xi)| \leq m^{2} / 8,000$. Then

$$
|D(\xi)| \geq \frac{R^{2}}{15}
$$

Proposition 8.3. Assume that $\left|\mathscr{S}\left(\xi_{0}\right)\right| \geq \varepsilon m^{2}$ for some $\varepsilon>0$, and that $\varepsilon \mathrm{m}^{2}$ is large relative to $1 / \delta$. Then, for appropriate $C_{8}>0$,

$$
P\left(\left|\mathscr{S}\left(\xi_{t}\right)\right| \leq \frac{\varepsilon m^{2}}{2} \text { at some } t \leq \varepsilon_{2}\right) \leq \exp \left\{-C_{8} \varepsilon m^{2}\right\},
$$

where $\varepsilon_{2}=\left(16 \delta R^{2}\right)^{-1}$. Assume further that $\left|D\left(\xi_{0}\right)\right| \geq \varepsilon^{\prime} R^{2}$ for some $0<$ $\varepsilon^{\prime} \leq 1 / 10$ and that $\varepsilon^{\prime}\left(m^{2} \wedge N^{2}\right)$ is large relative to $1 / \delta$. Then

$$
P\left(K\left(\xi_{\varepsilon_{2}}\right) \leq \frac{N^{2}}{20} \text { or }\left|D\left(\xi_{\varepsilon_{2}}\right)\right| \leq \frac{\varepsilon^{\prime} R^{2}}{2}\right) \leq \exp \left\{-\frac{C_{8} \varepsilon \varepsilon^{\prime}}{\delta}\right\}
$$

For $\lambda \leq \delta m^{2}$, we defined $B$ to be occupied (well occupied) for the configuration $\xi$ if conditions (1)-(3) before Proposition 6.6 were satisfied. For $\lambda>$ $\delta m^{2}$, we now define $B$ to be occupied if either (1) $K(\xi) \geq \varepsilon_{4}\left(m^{2} \wedge N^{2}\right)$ or (2) $|\mathscr{S}(\xi)| \geq \varepsilon_{5} m^{2}$, and well occupied if the constants are replaced by $\varepsilon_{4}^{\prime}$ and $\varepsilon_{5}^{\prime}$. We set $\varepsilon_{4}^{\prime}=4 \varepsilon_{4}=2 \varepsilon_{1}=1 / 4,000\left(\varepsilon_{1}\right.$ was defined in Section 6), and $\varepsilon_{5}^{\prime}=2^{j_{1}} \varepsilon_{5}=\varepsilon_{1}=1 / 8,000$, where $j_{1}=3 \times 10^{6} / \delta$. (Needless to say, $\varepsilon_{5}$ is required to be quite small.) As in Section 6 , we use $\bar{\varepsilon}_{4}$ and $\bar{\varepsilon}_{5}$ to denote the bounds for occupied or well occupied squares, depending on the context. Condition (1) is the immediate equivalent of that for $\lambda \leq \delta m^{2}$; condition (2) on $|\mathscr{S}(\xi)|$ replaces that on $L(\xi)$. [For $\lambda \leq \delta m^{2}, \mathscr{S}(\xi)=\mathscr{P}(\xi)$.] We omit the third condition given for the case $\lambda \leq \delta m^{2}$, that $\xi(x)=1$ for some $x \in B$. A partial reason for this is that, under $\lambda>\delta m^{2}$, the transition from a configuration with $\xi(x)=1$ for some $x \in B$ would cause difficulties here, for example, the configurations with $\xi(x) \equiv 0$ for $x \in B$ are not occupied since $K(\xi)=0$ and $\mathscr{S}(\xi)=\varnothing$. As we shall see, configurations consisting of large numbers of monomers are not stable under $\lambda>\delta m^{2}$, which provides a physical justification for dropping the condition.

The main result in this section is the following proposition. 
Proposition 8.6. For small enough $\delta>0, \lambda>\delta m^{2}, T=1 / \delta$ and $m \wedge N$ large relative to $1 / \delta$,

$$
P(B(0) \text { is not occupied at some } t \in[T, 2 T]) \leq \delta
$$

if $B(z)$ is occupied at time 0 for some $z$ with $\|z\|_{1}=1$.

Proposition 8.6 is the analog of Proposition 6.6. As before, the multidimensional version given in (4.8) allows one to compare the process $V_{k}$ of occupied squares with the corresponding oriented percolation process $W_{k}$ by (4.10). When $\left|V_{0}\right|=\infty$, one employs (4.10) and continues with the same reasoning as in Sections 5 and 6 to show that monomers survive for the case $\lambda>\delta m^{2}$ as well. We are only assuming $\xi_{0} \in \Xi_{1}$ for the initial state, however. This allows the possibility here of only a finite number of occupied squares $B(z)$ initially. [This is the price one pays for excluding condition (3) for occupied sites in Section 6 from the conditions for $\lambda>\delta m^{2}$.] To bridge this gap, one notes the following. Let $\Xi_{3}$ denote those configurations where there is at least one $N \times N$ square not containing any monomer. For $\xi_{t} \in \Xi_{3}^{c}$, there will never again be any polymer atoms on $\mathbf{Z}^{2}$, and the mean density of vacant sites will drop off exponentially quickly. So, monomer poisoning will occur in this case. In order to show that monomers survive under $\Xi_{1}$, it therefore suffices to show that, on the set $\left\{\xi_{t} \in \Xi_{3}\right.$ for all $\left.t\right\}$, the probability of monomer survival is as close to 1 as desired.

We show this by using a fairly standard restarting argument. Assume that $\xi_{t_{0}} \in \Xi_{3}$, where $t_{0}=j(l T+1)$ and $j, l \in \mathbf{Z}^{+}$, with $l$ being regarded as fixed. With a fixed probability $\varepsilon>0$, some square $B(z), z=z j$, will be occupied at time $t_{0}+1$. The process $V_{k}^{j}$, which corresponds to $V_{k}$, but where time is shifted by $t_{0}+1$, will dominate the corresponding process $W_{k}^{j}$ as in (4.10). Hence,

$$
W_{k}^{\{z\}, j \subset V_{k}^{j},}
$$

where $W_{k}^{\{z\}, j}$ is the restriction of $W_{k}^{j}$ given by $W_{0}^{\{z\}, j}=\{z\}$. By Lemma 4.4, the evolution of $W_{k}^{\{z\}, j}$ ensures survival of monomers on the set $\Omega_{\infty}^{\{z\}, j}$.

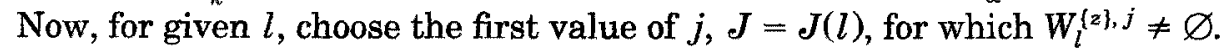
By the Markov property, the waiting time for this event has an exponential tail in $j$. One has, for any $l$, that

$$
\Omega_{\infty}^{\{z\}, J} \subset\left\{W_{l}^{\{z\}, J} \neq \varnothing\right\} .
$$

Also,

$$
\lim _{l \rightarrow \infty} P\left(\left(\Omega_{\infty}^{\{z\}, J}\right)^{c}\right)=\lim _{l \rightarrow \infty} P\left(\left(\Omega_{\infty}^{\{0\}}\right)^{c} \mid W_{l}^{[0]} \neq \varnothing\right)=0 .
$$

So, one can choose $l$ to make the probability in (8.8) as small as desired. It

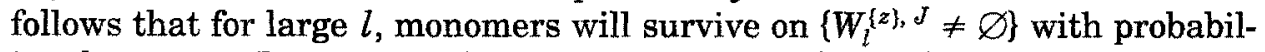
ity close to 1 . The same is therefore also true on $\left\{\xi_{t} \in \Xi_{3}\right.$ for all $\left.t\right\}$, which is the desired conclusion. 
Demonstration of Proposition 8.6. We proceed along the lines of the argument given in Section 6, starting with (6.23). As in (6.23) and (6.24), we introduce the stopping time $S_{0}^{1}$ and sets $\hat{F}_{1}$ and $\hat{F}_{2}$, where

$$
S_{0}^{1}=\inf \left\{t: K\left(\xi_{t}\right) \leq \bar{\varepsilon}_{4}\left(m^{2} \wedge N^{2}\right) \text { and }\left|\mathscr{S}\left(\xi_{t}\right)\right| \leq \bar{\varepsilon}_{5} m^{2}\right\}
$$

and

$$
\begin{aligned}
& \hat{F}_{1}=\left\{\xi: K(\xi)=\left\lfloor\bar{\varepsilon}_{4}\left(m^{2} \wedge N^{2}\right)\right\rfloor \text { and }|\mathscr{S}(\xi)| \leq\left\lfloor\bar{\varepsilon}_{5} m^{2}\right\rfloor\right\} \\
& \hat{F}_{2}=\left\{\xi: K(\xi)<\left\lfloor\bar{\varepsilon}_{4}\left(m^{2} \wedge N^{2}\right)\right\rfloor \text { and }|\mathscr{S}(\xi)|=\left\lfloor\bar{\varepsilon}_{5} m^{2}\right\rfloor\right\} .
\end{aligned}
$$

Note that $S_{0}^{1}$ stops the process $\xi_{t}$ when conditions (1) and (2) run into trouble. Since $K\left(\xi_{t}\right)$ and $\left|\mathscr{S}\left(\xi_{t}\right)\right|$ only decrease in increments of 1 , it follows that for $B$ occupied or well occupied under $\xi_{0}$,

$$
\xi_{S_{0}^{1}} \in \hat{F}_{1} \cup \hat{F}_{2} \text { on } S_{0}^{1}<\infty,
$$

which is the analog of (6.25). Note that the situation for (8.11) is, however, somewhat different than for (6.25), since for $\lambda \leq \delta m^{2}, B$ was occupied if it contained monomers, but that this is not true here.

Instead of working directly with $\hat{F}_{1}$ and $\hat{F}_{2}$, we will consider the sets

$$
\begin{aligned}
& F_{11}=\left\{\xi: K(\xi)=\left\lfloor\bar{\varepsilon}_{4}\left(m^{2} \wedge N^{2}\right)\right],|D(\xi)| \geq R^{2} / 15\right\}, \\
& F_{12}=\left\{\xi: K(\xi)=\left\lfloor\bar{\varepsilon}_{4}\left(m^{2} \wedge N^{2}\right)\right\rfloor,|\mathscr{Q}(\xi)| \geq \varepsilon_{6} m^{2}\right\} \cap F_{11}^{c}, \\
& F_{21}=\left\{\xi:|\mathscr{S}(\xi)|=\left\lfloor\bar{\varepsilon}_{5} m^{2}\right],|D(\xi)| \geq R^{2} / 15\right\} \cap F_{11}^{c} \cap F_{12}^{c}, \\
& F_{22}=\left\{\xi:|\mathscr{S}(\xi)|=\left[\bar{\varepsilon}_{5} m^{2}\right\rfloor,|\mathscr{Q}(\xi)| \geq \varepsilon_{6} m^{2}\right\} \cap F_{11}^{c} \cap F_{12}^{c} \cap F_{21}^{c},
\end{aligned}
$$

where $\varepsilon_{6}=1 / 8,000$. By Lemma 8.1 , under $\hat{F}_{i}, i=1,2$, either $|D(\xi)| \geq R^{2} / 15$ or $|\mathscr{Q}(\xi)| \geq \varepsilon_{6} m^{2}$. So,

$$
\hat{F}_{1} \subset F_{11} \cup F_{12} \text { and } \hat{F}_{2} \subset F_{21} \cup F_{22} .
$$

Together with (8.11), this gives

$$
\xi_{S_{0}^{1}} \in \bigcup_{i, j=1}^{2} F_{i j} \text { on } S_{0}^{1}<\infty
$$

for $B$ occupied or well occupied under $\xi_{0}$. The sets $\hat{F}_{1}$ and $\hat{F}_{2}$ should be thought of as the analogs of $F_{1}$ and $F_{2}$ in Section 6. Since $\mathscr{S}(\xi) \neq \mathscr{P}(\xi)$ in general for $\lambda>\delta m^{2}$, each case splits up into the two subcases, $F_{i 1}$ and $F_{i 2}$. The subcases $F_{11}$ and $F_{21}$ can be handled analogously to $F_{1}$ and $F_{2}$ in Section 6 , whereas we need to modify our reasoning for $F_{12}$ and $F_{22}$.

The goal in all four cases is to stop $\xi_{t}$ at a later time $\hat{S}$ at which $K\left(\xi_{\hat{S}}\right)$ and $D\left(\xi_{\hat{S}}\right)$ are large enough to apply Proposition 6.3. Choosing $\varepsilon=1 / 30$, as before, will then allow us to control the behavior of $\xi_{t}$ on the comparatively long interval $[\hat{S}, \hat{\sigma}]$, where $\hat{\sigma}=\left(\hat{S}+\varepsilon_{3}\right) \wedge(2 / \delta)$ and $\varepsilon_{3}=1 /\left(3 \times 10^{6}\right)$. Together with bounds for $\xi_{t}$ over $[0, \hat{S}]$, this allows us to control $\xi_{t}$ over $[0, \hat{\sigma}]$. As in Section 6, one can then iterate this procedure for $\xi_{t}$ over $[0,2 / \delta]$. 
We set $\hat{S}=S_{0}^{1}$ for $\xi_{S_{0}^{1}} \in F_{11}$, since the conditions on $K$ and $D$ are already satisfied in this case. For $\xi_{S_{0}^{1}} \in F_{21}$, we need to first apply (8.5) of Proposition 8.3 , with $\varepsilon=\bar{\varepsilon}_{5}$ and $\varepsilon^{\prime}=1 / 15$, together with the strong Markov property, to obtain the analog of (6.27), with $\hat{S}=S_{0}^{1}+\varepsilon_{2}$ and $\varepsilon_{2}=\left(16 \delta R^{2}\right)^{-1}$. [Inequality (8.4) controls the behavior of $\xi_{t}$ over $\left[S_{0}^{1}, \hat{S}\right]$.] Setting $\varepsilon=1 / 30$ and $a=\left[\bar{\varepsilon}_{4}\left(m^{2} \wedge N^{2}\right)\right]$ in both cases, and applying Proposition 6.3, one obtains, for $\xi_{S_{0}^{1}} \in F_{11} \cup F_{21}$, bounds that correspond to (6.29) and (6.30) on the behavior of $\xi_{t}$ over $[\hat{S}, \hat{\sigma}]$. We replace (6.28), since we need to avoid the presence of monomers in $B$ as well as polymer poisoning for $\lambda>\delta m^{2}$. We instead employ the event $E$ that $B$ contains both a vacant site and a polymer atom. [Note that $\xi \in E$ ensures that monomers can bond in $B$, and that there are no monomers already present in any of the squares $B(z)$ with $\|z\|_{1} \leq 1$.] On $\xi_{S_{0}^{1}} \in F_{11} \cup F_{21},(6.28)-(6.30)$ then translate into the following bounds: for $\bar{\varepsilon}_{i}=\varepsilon_{i}, i=4,5$ and appropriate $C_{9}, C_{10}>0$,

$$
P\left(\xi_{t} \notin E \text { at some } t \in[\hat{S}, \hat{\sigma}] \mid \mathscr{F}_{\hat{S}}\right) \leq C_{9} \exp \left\{-C_{10}\left(m^{2} \wedge N^{2}\right)\right\},
$$

and for $\bar{\varepsilon}_{i}=\varepsilon_{i}^{\prime}$,

$$
\begin{aligned}
& P\left(K\left(\xi_{t}\right) \leq \varepsilon_{4}\left(m^{2} \wedge N^{2}\right) \text { at some } t \in[\hat{S}, \hat{\sigma}] \mid \mathscr{F}_{\hat{S}}\right) \\
& \quad \leq C_{9} \exp \left\{-C_{10}\left(m^{2} \wedge N^{2}\right)\right\} .
\end{aligned}
$$

For both values of $\bar{\varepsilon}_{i}$,

$$
P\left(K\left(\xi_{\hat{\sigma}}\right) \leq \bar{\varepsilon}_{4}\left(m^{2} \wedge N^{2}\right) \mid \mathscr{F}_{\hat{S}}\right) \leq C_{9} \exp \left\{-C_{10}\left(m^{2} \wedge N^{2}\right)\right\} .
$$

We wish to reduce the cases $F_{12}$ and $F_{22}$ to analogs of $F_{11}$ and $F_{21}$, which will then allow us to apply the above reasoning to $\xi_{S_{0}^{1}} \in F_{12} \cup F_{22}$ as well. For this, we will use the following lemmas on the behavior of $K$ and $\mathscr{Q}$.

LEMMA 8.17. Assume that $K\left(\xi_{0}\right) \geq K_{0}$ for a given $K_{0}$. Then, for $t \leq \varepsilon_{2}$,

$$
P\left(K\left(\xi_{t}\right) \leq K_{0}-1 / \delta\right) \leq \exp \left\{-\frac{c_{4}}{4 \delta}\right\} \text {. }
$$

LEMMA 8.19. Assume that $\mathscr{Q}\left(\xi_{0}\right) \geq Q_{0}$ for a given $Q_{0}$. Then, for $t \geq \varepsilon_{2}$,

$$
P\left(\left|\mathscr{Y}_{t}\left(\xi_{0}\right)\right| \leq \frac{Q_{0}}{8}\right) \leq \exp \left\{-\frac{c_{4} Q_{0}}{4}\right\} .
$$

Lemma 8.17 states that $K\left(\xi_{t}\right)$ decreases at most at a given rate; the estimate holds because monomers can bond in $\bar{B}$ at rate at most $7 R^{2}$. Lemma 8.19 gives a bound in the opposite direction for $\left|\mathscr{Y}_{t}\left(\xi_{0}\right)\right|$. This gives a lower bound on the number of polymers that have bonded in $B$ by a given time. The argument is analogous to that in Lemma 6.14. Both lemmas here use (5.10), which is the source of $c_{4}$. Lemma 8.17 will be used in Lemma 8.22 and Lemma 8.19 in Lemma 8.29.

Our basic approach for the cases $F_{12}$ and $F_{22}$ will be the following. Starting from either $\xi_{0} \in F_{12}$ or $\xi_{0} \in F_{22}$, over [0, $\left.\varepsilon_{2}\right]$, at least a fixed pro- 
portion of $B$ will be covered by polymer atoms (Lemma 8.19), with neither $K$ (Lemma 8.17) nor $\mathscr{S}[(8.4)$ of Proposition 8.3] decreasing by too much. A given fraction of these polymer atoms will be in $D$ at $t=\varepsilon_{2}$. Therefore, at $t=\varepsilon_{2}$, analogs of $F_{11}$ or $F_{21}$ hold, with the coefficients $\bar{\varepsilon}_{4}$ and $\bar{\varepsilon}_{5}$ being modified. Propositions 6.3 and 8.3 can then be applied.

A complication in this scenario is caused by the possible interaction of initially present monomers with the polymers when they bond, which could cause many of the associated polymer atoms to desorb in uncontrollable patterns. To handle this situation, we inductively define the following stopping times, $S_{j}^{1}$ and $S_{j}^{2}, j=0,1,2, \ldots$, starting from $S_{0}^{1}$ in (8.9), with

$$
\begin{array}{ll}
S_{j}^{1}=\inf \left\{t \geq S_{j-1}^{2}: K\left(\xi_{t}\right) \leq \bar{\varepsilon}_{4}\left(m^{2} \wedge N^{2}\right)-\frac{j}{\delta}\right. \\
\text { and } \left.\left|\mathscr{S}\left(\xi_{t}\right)\right| \leq 2^{-j} \bar{\varepsilon}_{5} m^{2}\right\}, \\
S_{j}^{2}=S_{j}^{1}+\varepsilon_{2} .
\end{array}
$$

The idea will be to continue along the sequence $\left\{S_{j}^{1}\right\}$ until these monomers are exhausted by bonding polymers, with the number of iterates required being at most $j_{0}=j_{1}-1=\left(3 \cdot 10^{6} / \delta\right)-1$. Although we only need to deal with $\xi_{S_{0}^{1}} \in F_{12} \cup F_{22}$ here, $F_{11}$ and $F_{21}$ are included as subcases under our assumption that $B$ is occupied or well occupied under $\xi_{0}$. The result we want, Proposition 8.36 , is given after two preliminary estimates.

We first note that, by repeatedly applying Lemma 8.17 and (8.4) over the intervals $\left[S_{j}^{1}, S_{j}^{2}\right]$, one can control the behavior of $K$ and $\mathscr{S}$ up to time $S_{j_{0}}^{1}$. (One uses $m \wedge N$ here instead of $m^{2} \wedge N^{2}$ as in Section 6 because of the factor $j_{0}$.)

LEMmA 8.22. Assume that $m \wedge N$ is large relative to $1 / \delta$. If $B$ is initially either occupied or well occupied, then

$$
\begin{aligned}
& P\left(K\left(\xi_{t}\right) \leq \frac{1}{2} \bar{\varepsilon}_{4}\left(m^{2} \wedge N^{2}\right),\left|\mathscr{S}\left(\xi_{t}\right)\right| \leq 2^{-j_{0}} \bar{\varepsilon}_{5} m^{2} \text { at some } t \in\left[0, S_{j_{0}}^{1}\right]\right) \\
& \quad \leq 2 j_{0} \exp \left\{-\frac{C_{11}}{\delta}\right\}
\end{aligned}
$$

for appropriate $C_{11}>0$.

Corollary 8.24 is an immediate consequence of Lemma 8.22 and our definitions of $\bar{\varepsilon}_{4}$ and $\bar{\varepsilon}_{5}$.

COROLlaRY 8.24. Assume that $m \wedge N$ is large relative to $1 / \delta$. If $B$ is initially occupied, then

$$
P\left(\xi_{t} \notin E \text { at some } t \in\left[0, S_{j_{0}}^{1}\right]\right) \leq 2 j_{0} \exp \left\{-\frac{C_{11}}{\delta}\right\},
$$


and if $B$ is initially well occupied, then

$$
P\left(B \text { is not occupied at some } t \in\left[0, S_{j_{0}}^{1}\right]\right) \leq 2 j_{0} \exp \left\{-\frac{C_{11}}{\delta}\right\} \text {. }
$$

We claim that $j_{0}$ is large enough so that $\left|D\left(\xi_{t}\right)\right|$ will typically be at least $R^{2} / 15$ sometime before $S_{j_{0}}^{1}$. For this, set

$$
J=\min \left\{j:\left|D\left(\xi_{S^{1}}\right)\right| \geq R^{2} / 15\right\} .
$$

We also let $Z_{3}(t)$ denote the number of polymers that have bonded completely inside $B$ by time $t$, and $Z_{4}(t)$ the number of polymer atoms in $B$ at time $t$. Recall that $Z_{1}(t)$ is the number of monomers that have bonded in $\bar{B}$ by time $t$ (where $\bar{B}=B_{M+2 R}^{0}$ consists of those sites within range $R$ of $B$ ). Since $7 R^{2}$ is an upper bound on the number of monomers initially in $\bar{B}$, one has the simple inequality

$$
Z_{4}(t) \geq N^{2} Z_{3}(t)-Z_{1}(t)-7 R^{2}
$$

for all $t$. Since $Z_{4}(t) \leq R^{2}$ trivially holds, one gets, for all $t$, that

$$
N^{2} Z_{3}(t)-Z_{1}(t) \leq 8 R^{2} .
$$

LEMMA 8.29. For any $\xi_{0}$ and appropriate $C_{12}>0$,

$$
P\left(J \geq j_{0} \text { and } S_{j_{0}}^{1} \leq \frac{2}{\delta}\right) \leq j_{1} \exp \left\{-C_{12} m^{2}\right\}
$$

ProOF. By Lemma 8.1 and (8.21), either

$$
\left|D\left(\xi_{S_{j}^{1}}\right)\right| \geq \frac{R^{2}}{15} \text { or }\left|\mathscr{Q}\left(\xi_{S_{j}^{1}}\right)\right| \geq \frac{m^{2}}{8,000}
$$

for any $j$. If the first alternative holds for some $j<j_{0}$, then automatically $J<j_{0}$. Denote by $G_{4}$ the event that the second alternative holds for all $j<j_{0}$, and set

$$
G_{5}=\left\{\left|\mathscr{Y}_{\varepsilon_{2}}\left(\xi_{S_{j}^{1}}\right)\right|>\frac{m^{2}}{64,000} \text { for all } j<j_{0}\right\} .
$$

By Lemma 8.19, with $Q_{0}=m^{2} / 8,000$ and $t=\varepsilon_{2}$,

$$
P\left(G_{4} \cap G_{5}^{c}\right) \leq j_{0} \exp \left\{-\frac{c_{4} m^{2}}{64,000}\right\} .
$$

Now, on $G_{5}$,

$$
Z_{3}\left(S_{j_{0}}^{1}\right) \geq \frac{m^{2} j_{0}}{64,000} \geq \frac{40}{\delta} m^{2}
$$


But the rate at which monomers bond in $\bar{B}$ is bounded by $7 R^{2}$, and so by (5.10),

$$
\begin{aligned}
P\left(Z_{1}\left(S_{j_{0}}^{1}\right) \geq \frac{28 R^{2}}{\delta}, S_{j_{0}}^{1} \leq \frac{2}{\delta}\right) & \leq P\left(Z_{1}\left(\frac{2}{\delta}\right) \geq \frac{28 R^{2}}{\delta}\right) \\
& \leq \exp \left\{-\frac{14 c_{4} R^{2}}{\delta}\right\} .
\end{aligned}
$$

The bounds given for $Z_{1}\left(S_{j_{0}}^{1}\right)$ and $Z_{3}\left(S_{j_{0}}^{1}\right)$ in (8.33) and (8.34) are inconsistent with (8.28). So,

$$
P\left(G_{5} \cap\left\{S_{j_{0}}^{1} \leq \frac{2}{\delta}\right\}\right) \leq \exp \left\{-\frac{14 c_{4} R^{2}}{\delta}\right\} .
$$

Together with (8.32), (8.35) shows that

$$
P\left(G_{4} \cap\left\{S_{j_{0}}^{1} \leq \frac{2}{\delta}\right\}\right) \leq j_{1} \exp \left\{-C_{12} m^{2}\right\}
$$

for appropriate $C_{12}>0$. This implies (8.30).

Combining Lemma 8.29 with Lemma 8.22 and Corollary 8.24 , we obtain the following behavior for $\xi_{t}$ over $\left[0, S_{J}^{1} \wedge(2 / \delta)\right]$.

Proposition 8.36. Assume that $m \wedge N$ is large relative to $1 / \delta$. If $B$ is initially occupied, then

$$
P\left(\xi_{t} \notin E \text { at some } t \in\left[0, S_{J}^{1} \wedge \frac{2}{\delta}\right]\right) \leq C_{13} \exp \left\{-\frac{C_{14}}{\delta}\right\},
$$

and if $B$ is initially well occupied, then

(8.38) $P\left(B\right.$ is not occupied at some $\left.t \in\left[0, S_{J}^{1} \wedge \frac{2}{\delta}\right]\right) \leq C_{13} \exp \left\{-\frac{C_{14}}{\delta}\right\}$ for appropriate $C_{13}$ and $C_{14}>0$. In both cases,

$$
\left|D\left(\xi_{S_{J}^{1}}\right)\right| \geq \frac{R^{2}}{15} \quad \text { on } J<\infty
$$

and

$$
\begin{aligned}
& P\left(K\left(\xi_{S_{J}^{1}}\right) \leq \frac{1}{2} \bar{\varepsilon}_{4}\left(m^{2} \wedge N^{2}\right),\left|\mathscr{S}\left(\xi_{S_{J}^{1}}\right)\right| \leq 2^{-j_{0}} \bar{\varepsilon}_{5} m^{2}, S_{J}^{1} \leq \frac{2}{\delta}\right) \\
& \quad \leq C_{13} \exp \left\{-\frac{C_{14}}{\delta}\right\} .
\end{aligned}
$$

Using Proposition 8.36, we are now able to control the behavior of $\xi_{t}$ over the interval $[0, \hat{\sigma}]$, where $\hat{\sigma}=\left(\hat{S}+\varepsilon_{3}\right) \wedge(2 / \delta)$ and $\hat{S}$ is to be prescribed. We give the following summary, which involves reasoning analogous to that in 
(6.26)-(6.30) and (8.13)-(8.16). By (8.39) and (8.40), we note that if $B$ is initially occupied or well occupied, then $\xi_{S^{1}}$, for $S_{J}^{1} \leq 2 / \delta$, typically belongs to one of the following analogs of $F_{11}$ and $F_{21}$ in (8.12):

$$
\begin{aligned}
& \tilde{F}_{11}=\left\{\xi: K(\xi)>\frac{1}{2} \bar{\varepsilon}_{4}\left(m^{2} \wedge N^{2}\right),|D(\xi)| \geq \frac{R^{2}}{15}\right\}, \\
& \tilde{F}_{21}=\left\{\xi:|\mathscr{S}(\xi)|>2^{-j_{0}} \bar{\varepsilon}_{5} m^{2},|D(\xi)| \geq \frac{R^{2}}{15}\right\} \cap \tilde{F}_{11}^{c} .
\end{aligned}
$$

For $\xi_{S_{J}^{1}} \in \tilde{F}_{11}$, we set $\hat{S}=S_{J}^{1}$. On $\hat{S}<2 / \delta$, we can then apply Proposition 6.3, with $\varepsilon=1 / 30$ and $a=\left\lfloor\bar{\varepsilon}_{4}\left(m^{2} \wedge N^{2}\right) / 2\right\rfloor$. (If $\hat{S} \geq 2 / \delta$, we can stop the procedure.) For $\xi_{S_{J}^{1}} \in \tilde{F}_{21}$, we apply Proposition 8.3 , with $\varepsilon=2^{-j_{0}} \bar{\varepsilon}_{5}$ and $\varepsilon^{\prime}=1 / 15$, to obtain the analog of (6.27), with $\hat{S}=S_{J}^{1}+\varepsilon_{2}$. (This small choice of $\varepsilon$ is reflected in the error bound and requires a correspondingly small choice of $\delta$.) We then apply Proposition 6.3, with $\varepsilon=1 / 30$ and $a=$ $\left\lfloor\bar{\varepsilon}_{4}\left(m^{2} \wedge N^{2}\right)\right\rfloor$. We get in both cases, as the reader can check, that if $B$ is initially occupied or well occupied, then (8.14)-(8.16) continue to hold for the same values of $\bar{\varepsilon}_{i}, i=4,5$. [The "extra factor of 2 " in $\varepsilon_{4}^{\prime}=4 \varepsilon_{4}$ is used in (8.15), and the coefficient 2 in (6.5) is used in (8.16) to compensate for the factor " $1 / 2$ " in the first half of (8.41).] The desired behavior of $\xi_{t}$, at $t \in[0, \hat{S}]$, also follows from our computations. The bounds (8.37) and (8.38) give us the behavior of $\xi_{t}$ over $[0, \hat{S}]$, for the case $\tilde{F}_{11}$. Together with (8.4), these bounds also give us the behavior over $[0, \hat{S}]$ for $\tilde{F}_{21}$. (The relationship $\varepsilon_{5}^{\prime}=2^{j_{1}} \varepsilon_{5}=2 \times 2^{j_{0}} \varepsilon_{5}$ is used to show that, under $\bar{\varepsilon}_{i}=\varepsilon_{i}^{\prime}, i=4,5, B$ typically remains occupied over $\left[S_{j}^{1}, \hat{S}\right]$ in the latter case.) These results can together be formulated as the following analog of Proposition 6.31.

PROPOSITION 8.42. Suppose that $m \wedge N$ is large relative to $1 / \delta$. If $B$ is initially occupied, then for appropriate $C_{15}$ and $C_{16}>0$,

$$
P\left(\xi_{t} \notin E \text { at some } t \in[0, \hat{\sigma}]\right) \leq C_{15} \exp \left\{-\frac{C_{16}}{\delta}\right\}
$$

and

$$
P(B \text { is not occupied at } \hat{\sigma}) \leq C_{15} \exp \left\{-\frac{C_{16}}{\delta}\right\} .
$$

If $B$ is initially well occupied, then

(8.45) $\quad P(B$ is not occupied at some $t \in[0, \hat{\sigma}]) \leq C_{15} \exp \left\{-\frac{C_{16}}{\delta}\right\}$

and

$$
P(B \text { is not well occupied at } \hat{\sigma}) \leq C_{15} \exp \left\{-\frac{C_{16}}{\delta}\right\}
$$


Proceeding as in Section 6, one can iterate the procedure given in Proposition 8.42 until time $2 / \delta$ is reached. This gives the following analog of Proposition 6.36.

Proposition 8.47. Suppose that $m \wedge N$ is large relative to $1 / \delta$. If $B$ is initially occupied, then for appropriate $C_{16}>0$ and $C_{17}$,

$$
P\left(\xi_{t} \notin E \text { at some } t \in\left[0, \frac{1}{\delta}\right]\right) \leq \frac{C_{17}}{\delta} \exp \left\{-\frac{C_{16}}{\delta}\right\} .
$$

If $B$ is initially well occupied, then

$$
P\left(B \text { is not occupied at some } t \in\left[0, \frac{2}{\delta}\right]\right) \leq \frac{C_{17}}{\delta} \exp \left\{-\frac{C_{16}}{\delta}\right\}
$$

We need one last lemma before demonstrating Proposition 8.6. Note that this is the first place we require the assumption that $\lambda>\delta m^{2}$ in our computations. The function $Y(t)$ denotes the Lebesgue measure of the set of times $s \in[0, t]$ where $\left|\mathscr{Q}\left(\xi_{s}\right)\right|>\varepsilon_{6} m^{2}\left(\varepsilon_{6}=1 / 8,000\right)$ and, as before, $Z_{1}(t)$ is the number of monomers that have bonded in $\bar{B}$ and $Z_{3}(t)$ is the number of polymers that have bonded in $B$ by time $t$.

LEMMA 8.50. Suppose that $\lambda>\delta m^{2}$ and that $m^{2}$ is large relative to $1 / \delta$. Then, for appropriate $C_{18}>0$,

$$
P\left(Y\left(\frac{1}{\delta}\right) \geq \frac{1}{2 \delta}\right) \leq 2 \exp \left\{-\frac{C_{18} R^{2}}{\delta}\right\} .
$$

Proof. Under $\left|\mathscr{Q}\left(\xi_{s}\right)\right|>\varepsilon_{6} m^{2}$, the rate at which polymers bond inside $B$ is greater than $4 \delta \varepsilon_{6} m^{2} R^{2}$. Setting

$$
\tilde{Z}_{3}(t)=Z_{3}\left(Y^{-1}(t)\right)
$$

it follows that $\tilde{Z}_{3}(t)$ dominates a Poisson process with this intensity. So, by (5.10),

$$
P\left(\tilde{Z}_{3}\left(\frac{1}{2 \delta}\right) \leq \varepsilon_{6} m^{2} R^{2}\right) \leq \exp \left\{-c_{4} \varepsilon_{6} m^{2} R^{2}\right\} .
$$

It follows, that

$$
P\left(Z_{3}\left(\frac{1}{\delta}\right) \leq \varepsilon_{6} m^{2} R^{2} \text { and } Y\left(\frac{1}{\delta}\right) \geq \frac{1}{2 \delta}\right) \leq \exp \left\{-c_{4} \varepsilon_{6} m^{2} R^{2}\right\} .
$$

On the other hand, since monomers bond in $\bar{B}$ at rate at most $7 R^{2}$,

$$
P\left(Z_{1}\left(\frac{1}{\delta}\right) \geq \frac{14 R^{2}}{\delta}\right) \leq \exp \left\{-\frac{7 c_{4} R^{2}}{\delta}\right\}
$$

by (5.10). The bounds given for $Z_{1}(1 / \delta)$ and $Z_{3}(1 / \delta)$ in (8.52) and (8.53) are inconsistent with (8.28) for $m^{2}$ large relative to $1 / \delta$. It therefore follows from 
(8.52) and (8.53), that for appropriate $C_{18}>0$,

$$
P\left(Y\left(\frac{1}{\delta}\right) \geq \frac{1}{2 \delta}\right) \leq 2 \exp \left\{-\frac{C_{18} R^{2}}{\delta}\right\} .
$$

[Note that if one wishes to couple different systems here along the lines of the discussion at the end of Section 4, then the coupling should be done over the time scale given by $Z_{3}(t)$.]

Using Proposition 8.47 and Lemma 8.50, we show Proposition 8.6.

ProOF OF PROPOSITION 8.6. If $B(z)$ is occupied at time 0 , the square contains polymer atoms for all $t \leq T=1 / \delta$, except on a set with probability given in (8.48). On the complement $G_{6}$ of this exceptional set, there can be no monomers in $B(0)$. Let $\mathscr{E}$ denote those configurations where either $B(0)$ is well occupied or $|D(\xi)| \geq R^{2} / 15$. It follows from Lemmas 8.1 and 8.50 , and the above observation, that

$$
P\left(\left|\left\{t \in\left[0, \frac{1}{\delta}\right]: \xi_{t} \in \mathscr{E}\right\}\right| \leq \frac{1}{2 \delta}\right) \leq \frac{2 C_{17}}{\delta} \exp \left\{-\frac{C_{16}}{\delta}\right\} .
$$

Denote the complement of the exceptional set in (8.54) by $G_{7}$.

On $G_{6}$, there is always a vacant site in $B(z)$ for $t \leq 1 / \delta$, since $\xi_{t} \in E$. So, when $\left|D\left(\xi_{t}\right)\right| \geq R^{2} / 15$, polymer atoms desorb from $D\left(\xi_{t}\right)$ at rate at least $\left(1 / 5 R^{2}\right)\left(R^{2} / 15\right)=1 / 75$. Let $\tau$ denote the first time $B(0)$ is well occupied. It follows from (8.54) and this observation, that, on the restriction $\{\tau>1 / \delta\} \cap$ $G_{7}$, the rate of desorption of polymer atoms from $D\left(\xi_{t}\right)$ up to time $1 / \delta$ is at least $1 / 75$ more than one-half of the time. It follows that

$$
P\left(\tau>\frac{1}{\delta}\right) \leq \frac{2 C_{17}}{\delta} \exp \left\{-\frac{C_{16}}{\delta}\right\}+\exp \left\{-\frac{1}{150 \delta}\right\}
$$

If one applies the strong Markov property at time $\tau$, one obtains that, over $[\tau, \tau+2 / \delta], B(0)$ is occupied off of an exceptional set whose probability is given in (8.49). So, if $B(z)$ is initially occupied,

$$
\begin{aligned}
& P(B(0) \text { is not occupied at some } t \in[T, 2 T]) \\
& \quad \leq \frac{3 C_{17}}{\delta} \exp \left\{-\frac{C_{16}}{\delta}\right\}+\exp \left\{-\frac{1}{150 \delta}\right\} .
\end{aligned}
$$

For sufficiently small $\delta>0$, it is clear that the right-hand side of (8.55) is dominated by $\delta$. This gives (8.7), and completes the proof of Proposition 8.6.

\section{REFERENCES}

Bezudinnhout, C. and Grimmetr, G. (1990). The critical contact process dies out. Ann. Probab. 18 1462-1482.

Bramson, M. (1989). Survival of nearest particle systems with low birth rate. Ann. Probab. 17 $433-443$.

Bramson, M. and DurRetT, R. (1988). A simple proof of the stability criterion of Gray and Griffeath. Probab. Theory Related Fields 80 293-298. 
Bramson, M., DurReTt, R. and Swindle, G. (1989). Statistical mechanics of crabgrass. Ann. Probab. 17 444-481.

Bramson, M. and Neuhauser, C. (1992). A catalytic surface reaction model. J. Comput. Appl. Math. 40 157-161.

Bramson, M. and NeUHAuser, C. (1993). Survival of one-dimensional cellular automata under random perturbations. Ann. Probab. 21 244-263.

DickMAN, R. (1986). Kinetic phase transition in a surface reaction model: mean field theory. Phys. Rev. A 34 4246-4250.

DurRetT, R. (1984). Oriented percolation in two dimensions. Ann. Probab. 12 999-1040.

DURRETT, R. (1991). A new method for proving the existence of phase transitions. In Spatial Stochastic Processes (K. S. Alexander and J. C. Watkins, eds.) 141-170. Birkhäuser, Boston.

DURRETT, R. (1992). Multicolor particle systems with large threshold and range. $J$. Theoret. Probab. 5 127-152.

DuRRETT, R. and NEUHAUSER, C. (1991). Epidemics with recovery. Ann. Appl. Probab. 1 189-206.

DuRretT, R. and NeUhauser, C. (1994). Particle systems and reaction-diffusion equations. Ann. Probab. 22 289-333.

DURRETT, R. and SCHINAZI, R. (1993). Asymptotic critical value for a competition model. Ann. Appl. Probab. 3 1047-1066.

DURRETr, R. and SwINDLe, G. (1991). Are there bushes in the forest? Stochastic Process. Appl. 37 $19-31$.

DuRReTT, R. and SwINDLE, G. (1994). Coexistence results for catalysts. Probab. Theory Related Fields $98489-515$.

ENGEL, T. and ERTL, G. (1979). Elementary steps in the catalytic oxidation of carbon monoxide in platinum metals. Advances in Catalysis 28 1-63.

Evans, J. and MiEsch, M. (1991). Characterizing kinetics near a first-order catalytic poisoning transition. Phys. Rev. Lett. 66 833-836.

Jensen, I., Fogedby, H. and DickMan, R. (1990). Critical exponents for an irreversible surface reaction model. Phys. Rev. A 41 3411-3414.

Grannan, E. and Swindle, G. (1990). Rigorous results on a mathematical model of catalytic surfaces. J. Statist. Phys. 61 1085-1103.

LIGGETT, T. M. (1985). Interacting Particle Systems. Springer, New York.

LigGetT, T. M., Schonmann, R. and STACEY, A. (1997). Domination by product measures. Ann. Probab. To appear.

MOUNTFord, T. and SUDBURY, A. (1992). An extension of a result of Grannan and Swindle on the poisoning of catalytic surfaces. J. Statist. Phys. 67 1219-1222.

ZIFF, R., GULARI, E. and BARSHAD, Y. (1986). Kinetic phase transition in an irreversible surface reaction model. Phys, Rev. Lett. 56 2553-2556.

Department of Mathematics

UNIVERSTTY OF WISCONSIN

VAN VleCk HaLL

480 LINCOLN DRIVE

MADISON, WisconsIN 53706

\author{
School of Mathematics \\ UNIVERSITY OF MINNESOTA \\ 127 VINCENT HALL \\ 206 Church STREet S.E. \\ MINNEAPOLIS, MiNNESOTA 55455 \\ E-MAIL: nhauser@math.umn.edu
}

